\title{
Distinct multilevel misregulations of Parkin and PINK1 revealed in cell and animal models of TDP-43 proteinopathy
}

Xing Sun ${ }^{1,2}$, Yongjia Duan ${ }^{1,2}$, Caixia Qin ${ }^{3}$, Jian-Chiuan $\mathrm{Li}^{4}$, Gang Duan ${ }^{1,2}, \mathrm{Xue} \mathrm{Deng}^{1,2}$, Jiangxia Ni ${ }^{1,2}, \mathrm{Xu}_{\mathrm{CaO}}{ }^{1,2}$, Ke Xiang ${ }^{3}$, Kuili Tian ${ }^{1}$, Chun-Hong Chen ${ }^{4}$, Ang Li ${ }^{3}$ and Yanshan Fang $\mathbb{B D}^{1}$

\begin{abstract}
Parkin and PINK1 play an important role in mitochondrial quality control, whose malfunction may also be involved in the pathogenesis of amyotrophic lateral sclerosis (ALS). Excessive TDP-43 accumulation is a pathological hallmark of ALS and is associated with Parkin protein reduction in spinal cord neurons from sporadic ALS patients. In this study, we reveal that Parkin and PINK1 are differentially misregulated in TDP-43 proteinopathy at RNA and protein levels. Using knock-in flies, mouse primary neurons, and TDP- $43^{\mathrm{Q} 331 \mathrm{~K}}$ transgenic mice, we further unveil that TDP-43 downregulates Parkin mRNA, which involves an unidentified, intron-independent mechanism and requires the RNA-binding and the protein-protein interaction functions of TDP-43. Unlike Parkin, TDP-43 does not regulate PINK1 at an RNA level. Instead, excess of TDP-43 causes cytosolic accumulation of cleaved PINK1 due to impaired proteasomal activity, leading to compromised mitochondrial functions. Consistent with the alterations at the molecular and cellular levels, we show that transgenic upregulation of Parkin but downregulation of PINK1 suppresses TDP-43-induced degenerative phenotypes in a Drosophila model of ALS. Together, these findings highlight the challenge associated with the heterogeneity and complexity of ALS pathogenesis, while pointing to Parkin-PINK1 as a common pathway that may be differentially misregulated in TDP-43 proteinopathy.
\end{abstract}

\section{Introduction}

Amyotrophic lateral sclerosis (ALS) is an adult-onset neurodegenerative disease characterized by progressive motor neuron loss, leading to muscle weakness and wasting. ALS is incurable and the patients usually die within $2-5$ years after diagnosis. The majority $(>90 \%)$ of ALS cases are sporadic (sALS) with unknown causes, whereas mutations in genes such as SOD1, C9orf72, FUS, and TARDBP are reported to cause familial ALS that accounts for the remaining $10 \%^{1,2}$. Ubiquitin-positive

\footnotetext{
Correspondence: Ang Li (anglijnu@jnu.edu.cn) or Yanshan Fang (fangys@sioc. ac.cn)

${ }^{1}$ Interdisciplinary Research Center on Biology and Chemistry, Shanghai Institute of Organic Chemistry, Chinese Academy of Sciences, Shanghai 201210, China ${ }^{2}$ University of Chinese Academy of Sciences, Beijing 100049, China

Full list of author information is available at the end of the article.

These authors contributed equally: Xing Sun, Yongjia Duan

Edited by C. Munoz-Pinedo
}

cytoplasmic inclusions containing transactive response DNA-binding protein $43 \mathrm{kDa}$ (TDP-43, encoded by $T A R D B P$ ) in the brain and the spinal cord of patients are a main pathological hallmark of $\mathrm{ALS}^{3}$. Moreover, TDP-43positive protein inclusions are found in a large spectrum of neurodegenerative disorders, including frontotemporal lobar degeneration, Alzheimer's disease, dementia with Lewy bodies, polyglutamine diseases, and others ${ }^{4-8}$, which are collectively known as TDP-43 proteinopathy.

In physiological conditions, TDP-43 protein is predominantly localized to the nucleus. It belongs to the heterogeneous ribonucleoprotein family and plays an important role in regulating gene transcription, RNA processing, transport, and stability, as well as the formation of stress granules. In disease conditions, ubiquitinated TDP-43 accumulates in the cytoplasm. The

\section{(c) The Author(s) 2018}

(c) Open Access This article is licensed under a Creative Commons Attribution 4.0 International License, which permits use, sharing, adaptation, distribution and reproduction cc) in any medium or format, as long as you give appropriate credit to the original author(s) and the source, provide a link to the Creative Commons license, and indicate if changes were made. The images or other third party material in this article are included in the article's Creative Commons license, unless indicated otherwise in a credit line to the material. If material is not included in the article's Creative Commons license and your intended use is not permitted by statutory regulation or exceeds the permitted use, you will need to obtain permission directly from the copyright holder. To view a copy of this license, visit http://creativecommons.org/licenses/by/4.0/. 
mislocalization and aberrant aggregation of TDP-43 cause dysfunction of various aspects of RNA metabolism as well as protein homeostasis, eventually leading to motor neuron degeneration ${ }^{9-11}$. With the recent advancement of the next-generation sequencing, the RNA targets as well as the common principles of TDP-43mediated RNA regulations are emerging ${ }^{12-15}$.

The long intron-containing pre-mRNA of Parkin is one of the reported targets of TDP- $43^{13}$. Parkin is an E3 ubiquitin ligase involved in the clearance of damaged mitochondria via autophagy (termed "mitophagy"), which partners with PTEN-induced putative kinase 1 (PINK1) to execute the mitochondrial quality control function. PINK1 is a serine/threonine kinase, which after translation is continuously transported into mitochondria, cleaved, and released to the cytosol for proteasomemediated degradation ${ }^{16,17}$. When mitochondria are damaged, PINK1 cannot be effectively cleaved and is subsequently anchored on the mitochondrial outer membrane, which in turn recruits Parkin to mitochondria and induces mitophagy ${ }^{18}$. Mutations in Parkin and PINK1 genes are linked to autosomal recessive early-onset Parkinson's disease $(\mathrm{PD})^{19,20}$.

Increasing evidence points to mitochondrial dysfunction as a common pathogenic factor in $\mathrm{ALS}^{21,22}$. Interestingly, in the spinal cord autopsy samples from sALS patients, neurons with TDP-43 protein inclusions have reduced Parkin protein levels ${ }^{12}$. In this study, we investigate whether Parkin and PINK1 are involved in TDP43-induced neurodegeneration. We find that Parkin and PINK1 are differentially misregulated by TDP-43 at the RNA and protein levels. Consistently, genetic manipulations of Parkin or PINK1 exhibit the opposing modifying effects in a Drosophila model of TDP-43 proteinopathy. Collectively, we propose that distinct multilevel misregulations of Parkin and PINK1 contribute to the pathogenesis of TDP-43 proteinopathy.

\section{Results}

TDP-43 overexpression selectively decreases Parkin but not PINK1 mRNA levels

Previous studies showed that TDP-43 regulated Parkin pre-mRNA and TDP-43 loss of function (LOF) reduced Parkin mRNA levels in mouse brains ${ }^{12,13}$. Since TDP-43induced neurodegeneration involves both LOF and gainof-function (GOF) mechanisms ${ }^{23}$, in this study we investigated whether TDP-43 GOF affected Parkin and PINK1, starting with overexpressing human TDP-43 (hTDP-43) in mammalian systems. We examined the mRNA levels of Parkin and PINK1 in human 293T cells transfected with hTDP-43-HA and mouse primary neurons infected with lentivirus to express hTDP-43-HA (Fig. S1A). In both 293T cells (Fig. 1a) and primary mouse neurons (Fig. 1b), TDP-43 overexpression (OE) caused a significant reduction of Parkin mRNA levels compared to each of the control groups. However, in neither 293T cells (Fig. 1a) nor mouse neurons (Fig. 1b) did TDP-43 OE significantly alter PINK1 mRNA levels. Further, we found that in neurons derived from the transgenic mice expressing mutant hTDP-43 $3^{\mathrm{Q} 331 \mathrm{~K} 24}$ (Fig. S1B), Parkin mRNA and protein levels were also significantly decreased (Fig. 1c-e). Of note, the endogenous mouse TDP-43 protein level was decreased in hTDP- $43^{\mathrm{Q} 331 \mathrm{~K}}$-derived neurons, which was likely due to the inhibition of its own transcription by hTDP-43 OE as reported previously ${ }^{24}$. The total expression levels of both endogenous and exogenous TDP-43 proteins were examined in Fig. S1C-D.

To confirm this effect in an in vivo system, we examined mRNA levels of Parkin and PINK1 in the fly head of a previously established Drosophila ALS model of TDP- $43^{25,26}$. Consistently, we detected an almost $50 \%$ reduction of the Parkin mRNA levels in the hTDP-43 fly heads (Fig. 2a). In contrast, TDP-43 OE did not significantly affect PINK1 mRNA abundance $(p=0.565$, Fig. 2a). Hence, the mRNA levels of Parkin but not PINK1 were selectively reduced in multiple human cell, primary mouse neuron and in vivo fly models of TDP-43 proteinopathy.

\section{Generation of the dParkin-(HA) knock-in fly and examination of TDP-43 OE on Parkin protein levels in vivo}

Next, we sought to confirm the effect of TDP-43 OE on Parkin protein levels in the in vivo fly models. Unfortunately, none of the commercially available Parkin antibodies we have examined in this study worked in western blots with Drosophila Parkin protein (data not shown). To overcome this problem, we utilized the CRISPR-based gene editing and the transgenic Cas9-gRNA system ${ }^{27,28}$ to generate an HA knockin (KI) fly in which an HA-tag was inserted to the C terminus of the endogenous Drosophila Parkin (dParkin) gene (Fig S2A). Briefly, we constructed an HA donor vector flanked with dParkin sequences (pCR2TOPO-dParkin-2xHA-PBac-3xp3-eGFP) and two dParkin-stop codon guide RNA (gRNA) vectors (pBFvU6.3-dParkin-stop gRNA-1 and gRNA-2). The HA donor and the dParkin-stop gRNA plasmids were mixed for micro-injection using a nanos-Cas9 founder line. The desired transformants were selected for subsequent balancing and background clearing, and were eventually established as a stable dParkin-(HA) KI line (Fig. $2 b$ and Methods).

The 2xHA tag inserted to the $\mathrm{C}$ terminus of the endogenous dParkin gene allowed us to measure the endogenous Parkin protein levels by probing the HA-tag. Next, we crossed the dParkin-(HA) KI flies to hTDP-43 flies and examined the dParkin-HA protein levels by western 

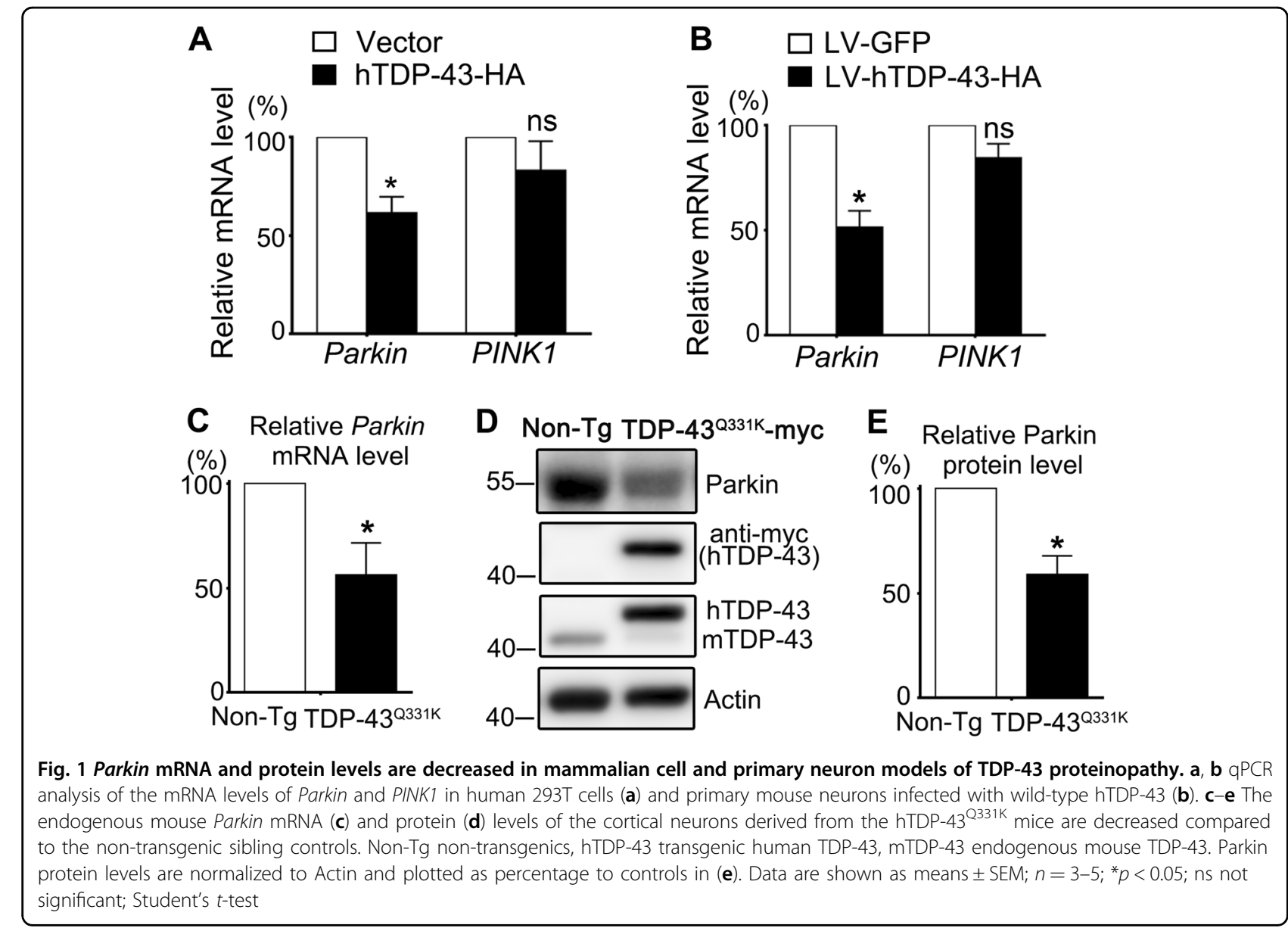

blotting. Compared to the control group (GMR driver only), expression of hTDP-43 in the fly eyes caused a significant reduction of the endogenous Parkin protein levels (Fig. 2c, d). Together, both the mRNA and protein levels of Parkin were decreased in the Drosophila model of TDP-43 proteinopathy.

\section{TDP-43 can decrease the mRNA and protein levels of}

\section{Parkin in the absence of intron/untranslated regions}

Previous RNA-seq studies indicated that TDP- 43 preferentially regulated pre-mRNAs with exceptionally long introns that are often more than $100 \mathrm{~kb}^{12-15}$. Human and mouse Parkin pre-mRNAs contained very long introns (Fig. S2B) and TDP-43 regulated their mRNA levels ${ }^{12,13}$. Intriguingly, we noticed that the dParkin gene contains only short introns (the longest $<200 \mathrm{bp}$, Fig. S2A). This raised the possibility that in addition to the reported intron-mediated regulation, TDP-43 might also regulate Parkin mRNA levels by an intronindependent mechanism. To test this hypothesis, we generated the transient expression plasmid that contains only the coding region of the human Parkin gene (FlagParkin, Fig. S2C).
We then examined whether TDP-43 could downregulate the mRNA levels of the plasmid-expressed, intron-free human Parkin, in mammalian cells. An intron-free PINK1 (PINK1-V5) constructed in the same expression plasmid was included as a control. The reverse transcription-PCR (RT-PCR) primers were designed to distinguish the Flag-tagged Parkin and V5-tagged PINK1 from the endogenously expressed Parkin and PINK1 mRNA in 293T cells (see Methods). As shown in Fig. 3a, b, the mRNA levels of Flag-Parkin were reduced by about $50 \%$ in TDP-43-overexpressing cells, whereas PINK1-V5 mRNA was not significantly changed (Fig. 3c, d). Consistently, the protein levels of the exogenous, intron-free Flag-Parkin were also drastically decreased by TDP-43 OE (Fig. 3e, f). In contrast, the protein levels of full-length (FL) PINK1 were not decreased, whereas cleaved PINK1 was increased (Fig. 3g, h; to be addressed later in this study). These data indicated that TDP-43 could regulate Parkin mRNA and protein levels in the absence of introns in mammalian cells, suggesting that TDP-43-mediated reduction of Parkin levels in sALS patients might involve both intron-based and intron-independent regulations of Parkin mRNA. Furthermore, since Parkin and PINK1 

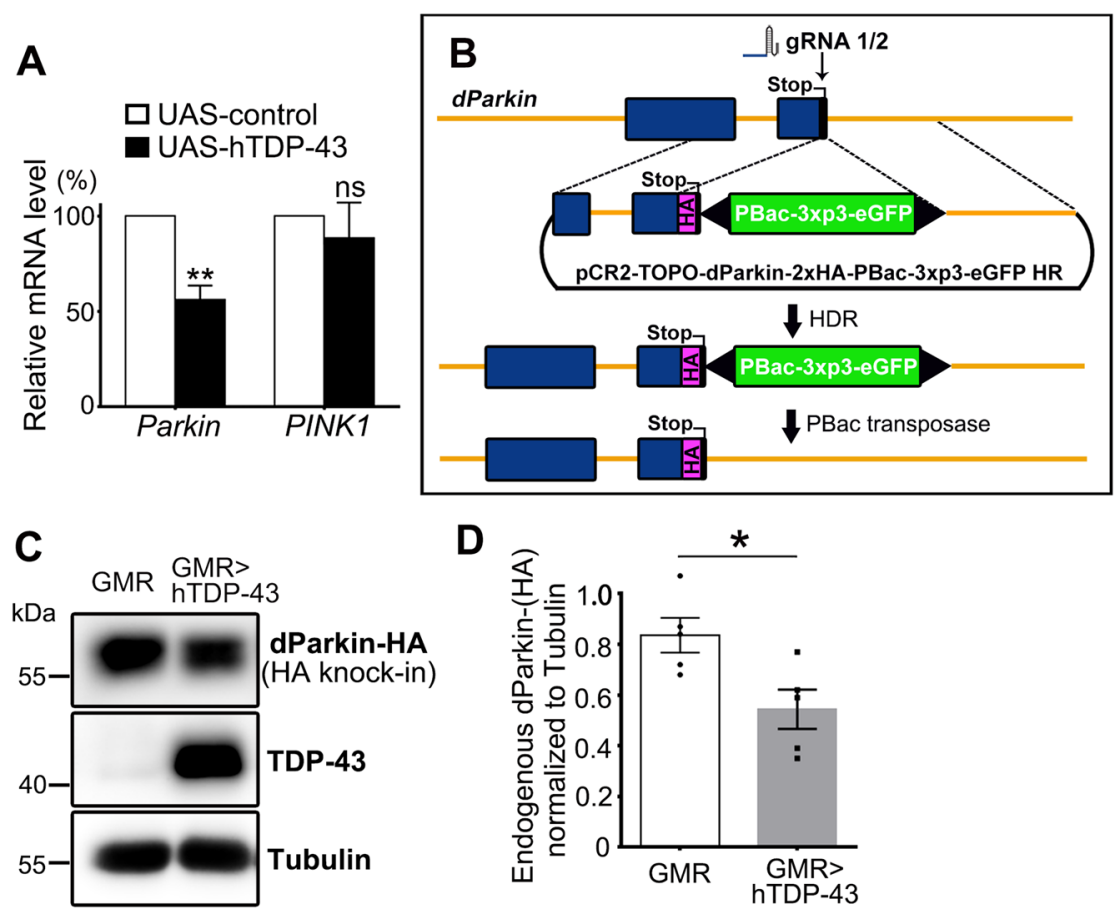

Fig. 2 Parkin mRNA and protein levels are reduced in the TDP-43 flies. a qPCR analysis of the mRNA levels of Parkin and PINK1 in the hTDP-43 fly heads (with a GMR-Gal4 driver). The mRNA levels of Parkin and PINK1 are normalized to actin and shown as average percentages to that of the control group. b A simplified overview of the process to generate the dParkin-(HA) knock-in flies using the transgenic Cas9-gRNA system (graph adapted from the flyCRISPR website). A pCR2-TOPO-dParkin-2xHA-PBac-3xp3-eGFP HR donor vector is generated to insert a 2xHA tag into the fly genome at the $C$ terminus of the dParkin gene by homology-directed repair (HDR). The successful knock-ins are identified by the GFP selection marker, which is subsequently removed by PBac transposase, leaving only the HA-tag and the stop codon in the target locus. c, $\mathbf{d}$ Western blot analysis of the endogenous Parkin protein levels measured by the knock-in HA-tag in the hTDP-43 flies or the driver only control (GMR) flies. The quantification is shown in $\mathbf{d}$. Data are means \pm SEM; $n=5 ;{ }^{*} p<0.05,{ }^{* *} p<0.01$; ns not significant; Student's $t$-test

were subcloned into the same vector with the same backbone and promoter (see Methods), it was unlikely that the selective downregulation of plasmid-expressed, intron-free Parkin was due to a general effect of TDP-43 on transcription or the untranslated regions (UTRs) of the expression plasmid.

\section{Downregulation of intron-free Parkin requires both the RRM1 and glycine-rich domains of TDP-43}

TDP-43 is a RNA-binding protein that consists of a nuclear localization signal and a nuclear export signal, two RNA recognition motif (RRM) motifs involved in DNA and RNA binding, and a glycine-rich domain (GRD) domain at the $\mathrm{C}$ terminus that mediates protein-protein interaction with other members of the heterogeneous nuclear RNP protein family ${ }^{9,10}$. To understand what motif (s) are required and how TDP-43 regulates Parkin in the absence of any intron or gene-specific UTR, we generated the plasmids to express truncated TDP-43 protein: hTDP43-HA- $\triangle R R M 1,-\triangle R R M 2$, and $-\triangle$ GRD (Fig. 4a). We found that hTDP-43- $\triangle$ RRM1 and hTDP-43- $\Delta$ GRD were unable to significantly downregulate the intronfree Parkin mRNA levels, whereas hTDP-43- $\triangle$ RRM2 showed a similar reduction of Parkin mRNA to that of the FL TDP-43 (Fig. 4b, c). Further, we examined the effects of FL and truncated TDP-43 on the protein levels of intron-free Parkin. Consistently, hTDP-43- $\Delta$ RRM1 and hTDP-43- $\triangle$ GRD could not downregulate the protein levels of Flag-Parkin, whereas hTDP-43- $\Delta$ RRM2 significantly decreased Flag-Parkin protein levels, and this was despite the fact that hTDP-43- $\Delta$ RRM2 was expressed at a lower level (Fig. $4 \mathrm{~d}$, e). These results suggest that the post-transcriptional, intron/UTR-independent downregulation of Parkin requires both the RNA-binding and protein-protein interaction functions of TDP-43.

\section{Increase of TDP-43 causes cytosolic accumulation of} cleaved PINK1 that forms insoluble aggregates in the cell

We noticed that although TDP-43 OE did not significantly change PINK1 mRNA or FL PINK1 protein levels $(\sim 64 \mathrm{kDa})$, there was a remarkable increase of cleaved PINK1 $\left(\sim 52 \mathrm{kDa}^{16,29}\right)$ in the western blots (Fig. 3g, h). Damaged mitochondria accumulate PINK1 on the outer membrane, which in turn recruits Parkin and induces mitophagy ${ }^{18}$. To determine if the TDP-43-induced increase of cleaved PINK1 was due to mitochondrial accumulation 

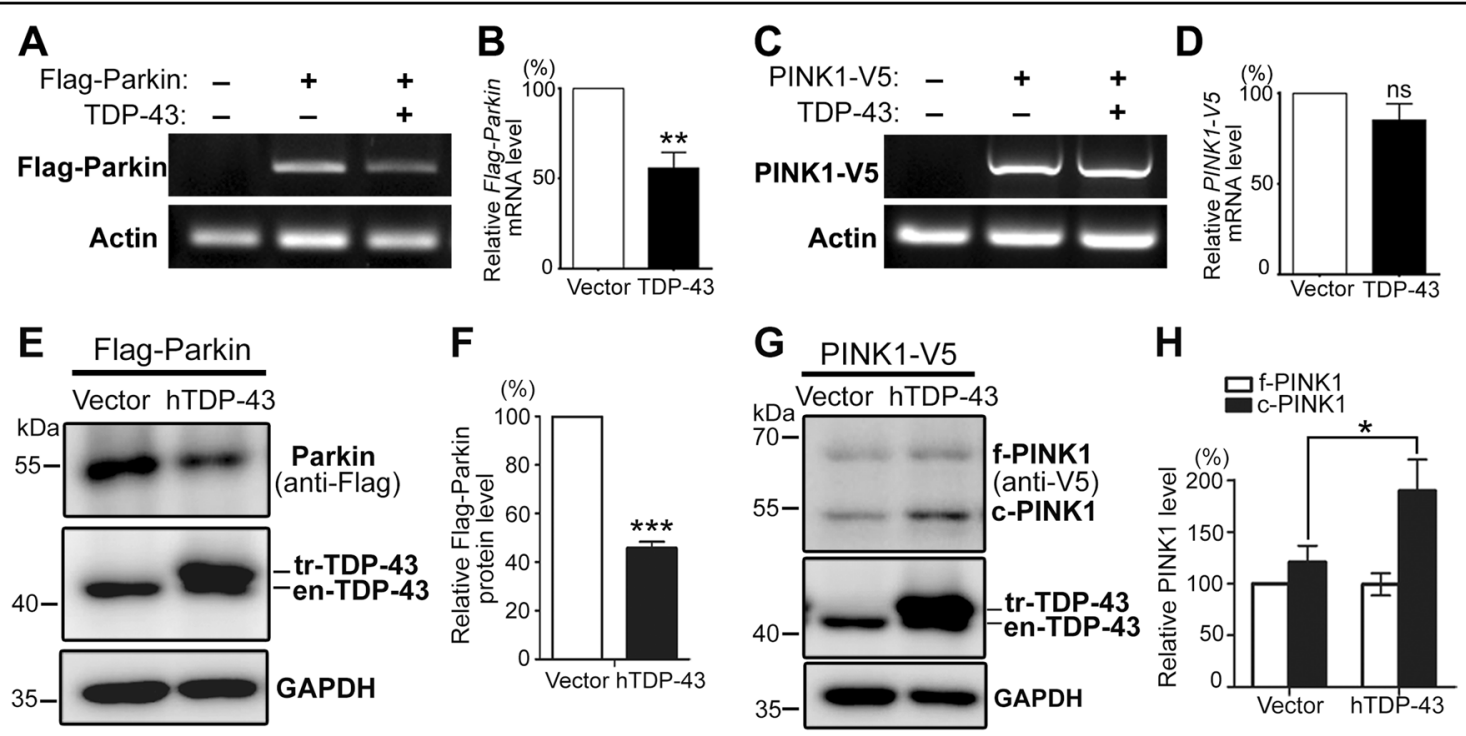

Fig. 3 TDP-43 OE can reduce the mRNA and protein levels of Parkin in an intron-independent manner. a-d TDP-43 selectively reduces the mRNA levels of plasmid-expressed, intron-free Parkin but not PINK1. The mRNA levels of transiently transfected Flag-Parkin (a) or PINK1-V5 (c) in 293T cells are evaluated by a semiquantitative RT-PCR assay, and quantified in $\mathbf{b}$ and $\mathbf{d}$, respectively. The specific PCR primers (containing the Flag or V5 tag sequence) are designed to distinguish the plasmid mRNAs from endogenously expressed Parkin and PINK1 mRNAs in 293T cells. e, f TDP-43 OE decreases the protein levels of Parkin but increases cleaved PINK1. Western blot analysis of protein levels of plasmid-expressed Flag-Parkin (e) or PINK1-V5 (g) in 293T cells, and quantified in $\mathbf{f}$ and $\mathbf{h}$, respectively. All protein levels are normalized to GAPDH. en-TDP-43 endogenous human TDP-43 in 293 T cells, tr-TDP-43 transfected hTDP-43-HA, f-PINK1 full-length PINK1, c-PINK1 cleaved PINK1. Data are means \pm SEM; $n=3-5 ;{ }^{*} p<0.05$, ${ }^{* *} p<0.01,{ }^{* * *} p<0.001$; ns not significant; Student's $t$-test

of PINK1, we examined the subcellular localization of PINK1 by immunocytochemistry. Since the expression of endogenous PINK1 protein was too low to be reproducibly detected in 293T cells, we transfected the cells with PINK1$\mathrm{V} 5$ and immunostained for the V5 tag. In the control cells, PINK1-V5 was predominantly co-localized with mitoDsRed (Fig. 5a), indicating that PINK1 was mostly localized to mitochondria under normal conditions. With TDP-43 OE, PINK1-V5 was no longer specifically localized to mitochondria but spread out in the cytoplasm (Fig. 5b). The reduced mitochondrial localization was further demonstrated by the co-localization analysis of PINK1-V5 and mito-DsRed (Fig. 5c, d).

In addition to the altered subcellular distribution of PINK1, another striking phenomenon we observed was that PINK1 formed massive puncta in the cells cotransfected with hTDP-43 (Fig. 5b, e). To determine if the PINK1 puncta were protein aggregates, we assessed the solubility of PINK1 by fractionation and western blot. In control cells, the FL PINK1 was mainly in the soluble fraction and the cleaved PINK1 was in the insoluble fraction. With TDP-43 OE, there was a robust increase of both the FL and cleaved PINK1 levels in the insoluble fraction, of which the cleaved PINK1 was more dramatically increased (Fig. 5f, g). In contrast, TDP43 did not significantly alter Parkin subcellular distribution or form insoluble aggregates (Fig. S3). Together, we concluded that TDP-43 OE led to a cytosolic accumulation of cleaved PINK1 that formed insoluble protein aggregates.

\section{PINK1 proteostasis is sensitive to TDP-43-induced proteasomal activity impairment}

In normal cells, cleaved PINK1 is released from mitochondria and undergoes rapid degradation in the cytoplasm via the ubiquitin proteasome system (UPS) $)^{17}$. As such, PINK1 protein usually is undetectable or at very low levels in healthy cells. The observation of TDP-43induced cytosolic accumulation of cleaved PINK1 (Fig. 5) strongly suggested that the function of UPS might be impaired in TDP-43 proteinopathy. To test this possibility, we measured the proteasomal activity of the cells using an in vitro fluorogenic peptide cleavage assay. Compared to the control cells, there was a small but significant reduction of proteasomal activity of the cells transfected with hTDP-43 (Fig. S4A). Considering that TDP-43-mediated neurodegeneration in diseases usually develops over years, we think a small decrease rather than an abrupt inhibtion of the proteasomal function may be more pathophysiologically relevent to the disease condition.

Since the reduction of proteasomal activity by TDP- 43 $\mathrm{OE}$ indicated by the in vitro assay was rather moderate, we asked if it was sufficient to significantly disturb 

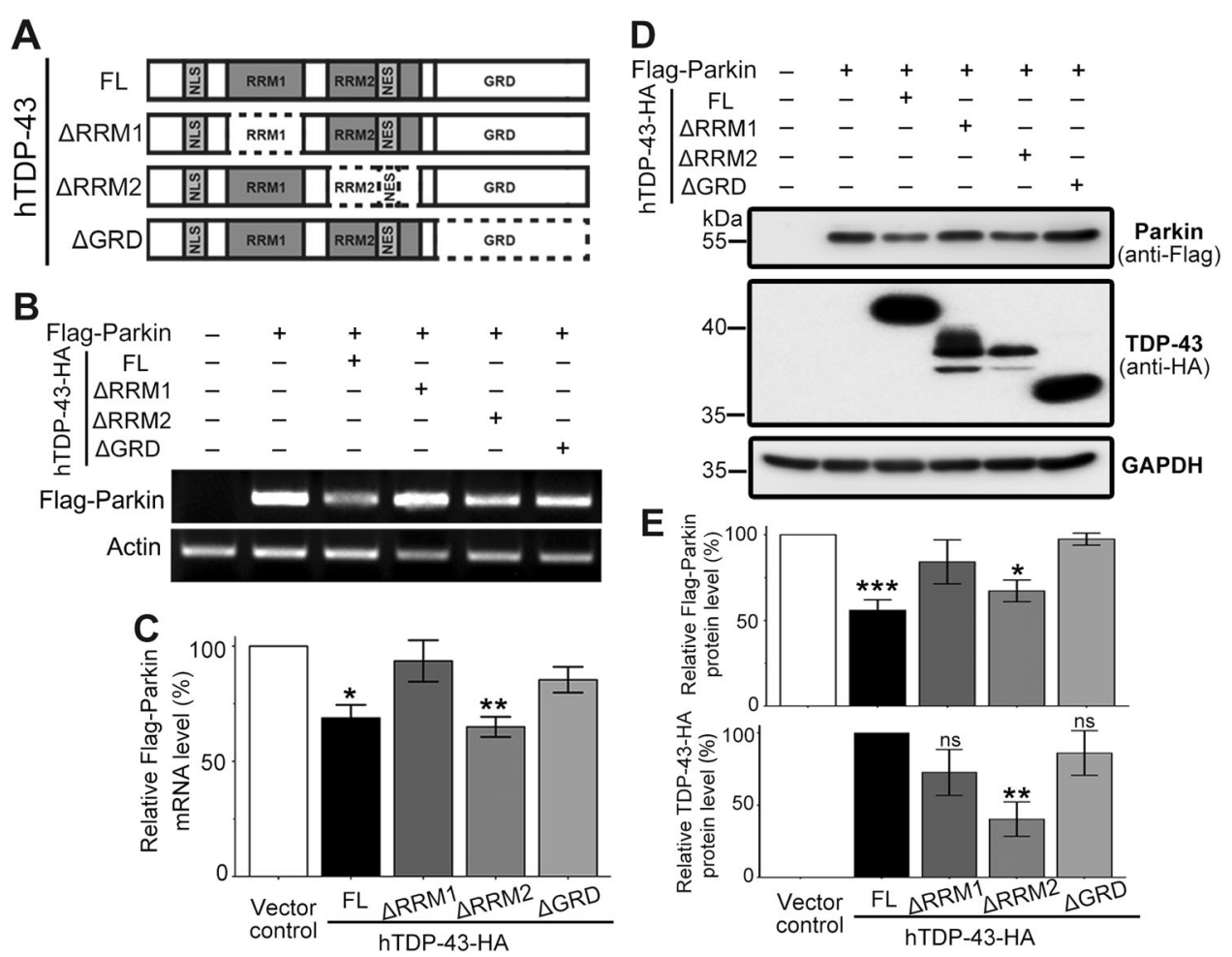

Fig. 4 The RRM1 and GRD domains of TDP-43 are required for downregulation of intron-free Parkin. a A schematic graph showing the major TDP-43 functional domains and the truncated TDP-43 proteins examined in this study. NLS nuclear localization signal, NES nuclear export signal, RRM RNA recognition motif, GRD glycine-rich domain. b-e Semiquantitative RT-PCR and western blotting analyses of mRNA (b) or protein levels (d) of plasmid-expressed, intron-free Flag-Parkin co-expressed with full-length (FL) or truncated hTDP-43-HA as indicated. The PCR primers (containing the Flag tag sequence) are designed to only amplify the plasmid-expressed Flag-Parkin but not the endogenous Parkin, confirmed by the negative control. The quantifications of $\mathbf{b}$ and $\mathbf{d}$ were shown in $\mathbf{c}$ and $\mathbf{e}$, respectively. The relative mRNA and protein levels of Flag-Parkin in the vector control group and the protein level of TDP-43 in the hTDP-43-FL group are set to $100 \%$. Data are means \pm SEM; $n=$ of $3-5 ;{ }^{*} p<0.05,{ }^{* *} p<0.01$, ${ }^{* * *} p<0.001$; ns not significant; one-way ANOVA

the proteostasis of PINK1. To address this question, we treated the $293 \mathrm{~T}$ cells with the proteasome inhibitor MG-132 at a series of concentrations, ranging from $5 \mathrm{nM}$ to $5 \mu \mathrm{M}$ for $3 \mathrm{~h}$ (Fig. S4B). The concentration of $50 \mathrm{nM}$ generated a mild inhibition that is similar to the effect of hTDP-43 OE in 293T cells (Fig. S4A). We then treated the cells with MG-132 using this condition. Similar to hTDP-43 OE, no increase of Parkin intensity or Parkin protein aggregate was observed at this condition (Fig. S4C-D, G). In contrast, the same treatment of MG-132 inhibition caused a robust increase of PINK1 overall intensity and a massive accumulation of PINK1 aggregates in the cytoplasm (Fig. S4E-F, H). This result was consistent with the effects of TDP-43 OE on Parkin (Fig. S3) and PINK1 (Fig. 5), indicating that the turnover of PINK1 protein was extremely sensitive to alterations of proteasomal activity. Hence, TDP-43-induced mild impairment of proteasomal activity selectively hindered the turnover of proteasome function-sensitive proteins such as PINK1, but not other less labile proteins such as Parkin.
This gave rise to the distinct effects that TDP-43 OE downregulated Parkin levels but led to a cytosolic accumulation of PINK1. Thus, in addition to intronbased regulation of Parkin pre-mRNA ${ }^{12,13}$ and intronindependent regulation of Parkin mRNA (Figs. 3 and 4), a third level of misregulation was on PINK1 protein turnover due to impaired proteasomal activity in TDP- 43 proteinopathy.

\section{Endogenous PINK1 protein accumulates in TDP-43 ${ }^{\mathrm{Q331K}}$ mice}

To confirm if endogenous PINK1 was similarly misregulated by TDP-43 in an in vivo mammalian model, we examined the PINK1 protein levels in the motor cortex of the 8-month-old TDP- $43^{\mathrm{Q} 331 \mathrm{~K}}$ mice. Consistently, we observed a remarkable increase of both $\mathrm{FL}$ and cleaved PINK1 proteins compared to the non-transgenic sibling control mice (Fig. 6a, b). As mentioned earlier, PINK1 protein is usually rapidly turned over and has a very low basal level in normal cells ${ }^{17}$. The accumulation of PINK1 protein in TDP-43 neurons is likely to be deleterious. 

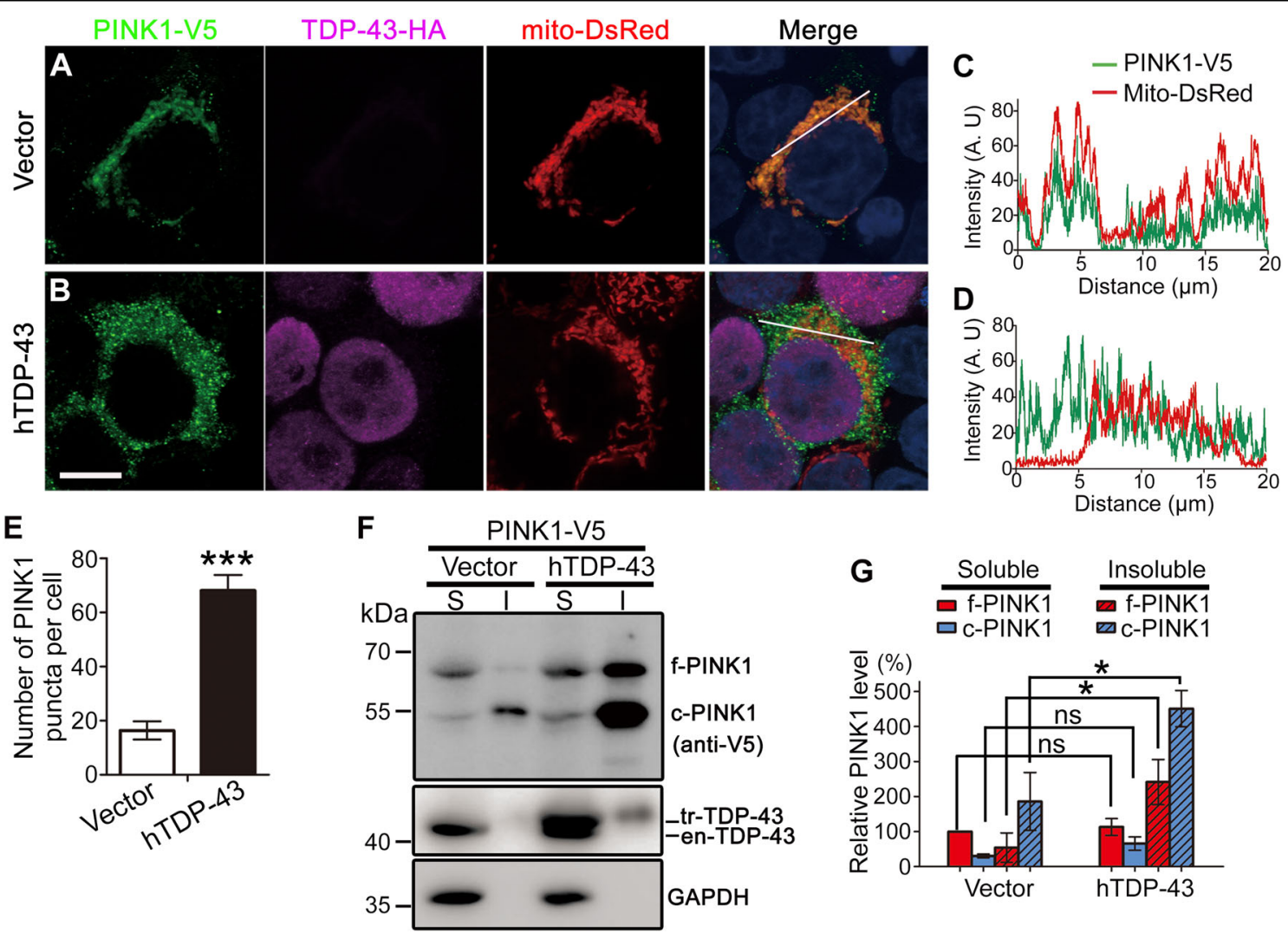

Fig. 5 TDP-43 OE promotes cytosolic accumulation of cleaved PINK1 that forms insoluble aggregates. a, d 293T cells transiently transfected with PINK1-V5 in the absence (vector control) (a) or presence of co-transfection of hTDP-43-HA (b). Representative confocal images show cells immunostained for PINK1 (V5) and TDP-43 (HA). Mitochondria are labeled by mito-DsRed and a merge of all channels is shown with the nucleus stained by DAPI in blue. The co-localization of PINK1 with mitochondria (mito-DsRed) is evaluated by the line scanning analysis in $\mathbf{c}$ and $\mathbf{d}$. e The number of protein puncta is counted using the "Analyze Particles" module of ImageJ and shown as average numbers of puncta per cell. Data are means \pm SEM of $\sim 100$ cells per group; ${ }^{* * *} p<0.001$. Scale bar: $10 \mu \mathrm{m}$. f, g 293T cells transfected of PINK1-V5 together with empty vector (control) or hTDP-43 are lysed and fractionated. The RIPA-soluble and -insoluble (resolved in $9 \mathrm{M}$ urea) fractions are analyzed by western blotting (f) and quantified in $\mathbf{g}$. S soluble fraction, I insoluble fraction, en-TDP-43 endogenous TDP-43, tr-TDP-43 transfected hTDP-43-HA, f-PINK1 full-length PINK1, c-PINK1 cleaved PINK1. All protein levels are normalized to GAPDH. The relative protein level of soluble full-length PINK1 in the control group (vector) is set to $100 \%$. Data are means \pm SEM; $n=3$; ${ }^{*} p<0.05$; Student's $t$-test; ns not significant

Consistent with this idea, we observed that neuronal upregulation of PINK1 reduced the Drosophila lifespan by $\sim 6.9 \%$ (Fig. S5A-B).

\section{Increase of cleaved PINK1 reduces mitochondrial functions}

To further elucidate the cellular consequence of PINK1 accumulation, especially the increase of cleaved PINK1, we generated the plasmid to express truncated PINK1 (PINK1 ${ }^{\Delta 1-104}-$ V5, Fig. 6c, d) that mimicked the protein product after the FL PINK1 was cleaved ${ }^{17}$. We used a Seahorse XFe96 Analyzer together with the Cell Mito Stress Test to measure the oxygen consumption rate (OCR) of the 293 T cells transfected with PINK1 ${ }^{\Delta 1-104}$ V5 or vector. The basal mitochondrial respiration occurred similarly, whereas the spare respiratory capacity was significantly reduced in PINK1 ${ }^{\Delta 1-104}$-V5 cells (Fig. 6e); the maximal respiration also showed a trend to decrease although not statistically significant ( $p=0.066$; Fig. 6e). The data suggest that under normal conditions, cells with accumulation of cleaved PINK1 may function normally. However, under stress, these cells can exhibit reduced mitochondrial activity, which over years in aging might promote the pathogenesis of neurodegeneration in disease.

Upregulation of Parkin, whereas downregulation of PINK1 improves the degenerative phenotypes of TDP-43 flies

After revealing the multilevel misregulation of Parkin and PINK1 by TDP-43 OE at the cellular level, we were keen to know whether their misregulations contribute to ALS pathogenesis and whether restoring Parkin and PINK1 normal expression levels offers a means to ameliorate TDP-43-mediated neurodegeneration at the animal level. To answer these questions, we utilized an 

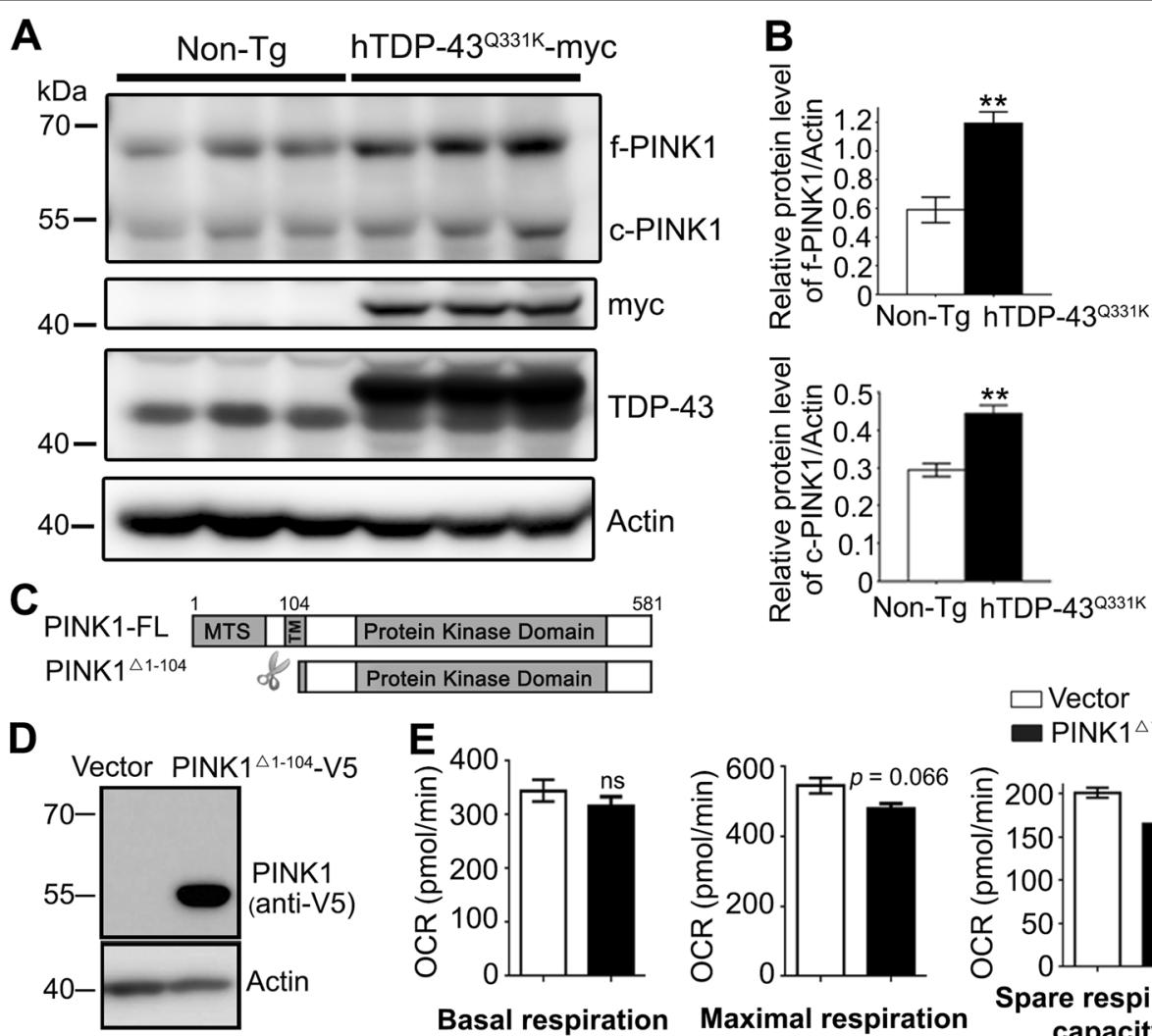

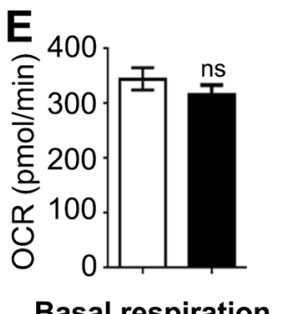

Basal respiration

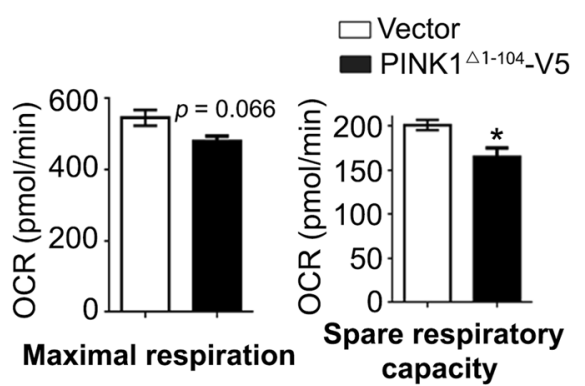

Fig. 6 Accumulation of PINK1 proteins in TDP-43 mice and compromised mitochondrial functions with cleaved PINK1. a, b The endogenous PINK1 protein levels in the motor cortex of 8-month-old hTDP-43 ${ }^{\mathrm{Q331K}}$ mice or age-matched non-transgenic controls are analyzed by western blotting in $\mathbf{a}$ and quantified in $\mathbf{b}$. Three mice of each genotype are tested. f-PINK1 full-length PINK1, c-PINK1 cleaved PINK1. All protein levels are normalized to actin. The relative expression levels of total TDP-43 proteins were examined in Fig. S1E. c A schematic drawing of the full-length PINK1FL and truncated PINK1 $\triangle^{1-104}$ after a cleavage at the amino acid 104. MTS mitochondrial targeting sequence, TM transmembrane domain. $\mathbf{d} A$ representative western blot confirming the PINK1 $\Delta^{1-104}-\mathrm{V} 5$ expression $(\sim 53 \mathrm{kDa})$ in $293 \mathrm{~T}$ cells. e The mitochondrial respiration functions in cells transfected with cleaved PINK1 are evaluated by the Seahorse XF Cell Mito Stress Test. The OCRs (pmol/min) of the basal, maximal respiration, and spare respiratory capacity are shown as means \pm SEM; $n=3 ;{ }^{*} p<0.05$ and ${ }^{* *} p<0.01$; ns not significant; Student's $t$-test

inducible elav-GeneSwich (elavGS) driver ${ }^{59}$ to express hTDP-43 in adult fly neurons only (induced after eclosion of the adult fly). Expression of hTDP-43 in adult fly neurons induced an age-dependent decline of climbing capability (see the control groups of RNAi- $m$ Cherry and UAS-lacZ in Fig. 7a, b). RNAi knockdown (KD) of PINK1 but not Parkin significantly delayed the age-dependent climbing deficits of hTDP-43 flies (D20 and D30, Fig. 7a), in spite of a more efficient KD of Parkin than that of PINK1 by the RNAi transgenes (Fig. S5C-D). On the contrary, Parkin OE provided remarkable suppression (D20 and D30, Fig. 7b), whereas PINK1 OE enhanced the age-dependent climbing deficits of hTDP-43 flies (D30, Fig. $7 \mathrm{~b}$ ). The KD efficiency and OE levels of the UAS transgenes are confirmed in Figure S5. Moreover, KD or OE of Parkin in fly neurons did not significantly change the protein levels or subcellular distribution of hTDP-43; nor did the genetic manipulations of PINK1 (Fig. S6). These data confirmed that the modifying effect of Parkin and PINK1 in the hTDP-43 flies was not due to an alteration of the TDP-43 protein per se.

Next, we evaluated the effects of manipulating Parkin and PINK1 expression levels on modifying the longevity of hTDP-43 flies. As shown in the lifespan experiments in Fig. 7c-e, the median lifespan of hTDP-43 flies was significantly increased by $11.2 \%$ with downregulation of PINK1 and by $14.8 \%$ with upregulation of Parkin in adult fly neurons. Considering that TDP-43 is a DNA/RNAbinding protein and excessive TDP-43 impairs proteasomal activity (Fig. S4), it is likely that there are other targets whose mRNA abundance and protein turnover are also affected. Thus, it is unsurprising that correcting the misregulations of Parkin or PINK1 only partially rescued the phenotypes of hTDP-43 flies. In an earlier study, we have shown that reduction of PINK1 levels in adult neurons of wild-type flies did not have a dramatic effect on aging ${ }^{30}$. And, although ubiquitous Parkin OE extended Drosophila lifespan ${ }^{31}$, neuronal OE of Parkin 

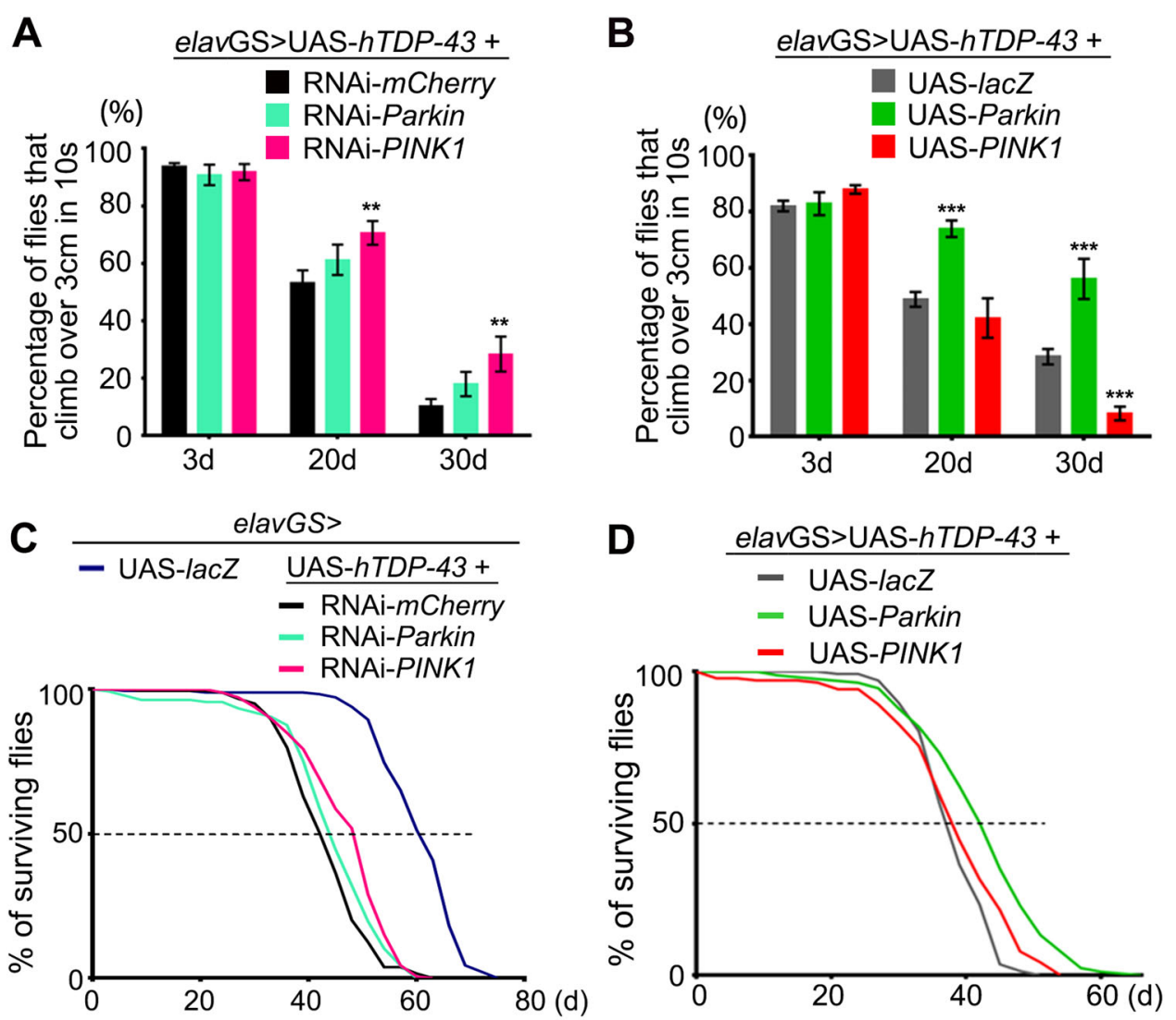

\begin{tabular}{|ccccc|}
\hline Eenotype & $\mathbf{n}$ & $\begin{array}{c}\text { Median lifespan } \\
\text { (d) }\end{array}$ & $\begin{array}{c}\text { Statistical } \\
\text { significance }\end{array}$ & $\begin{array}{c}\text { \% of median } \\
\text { lifespan change }\end{array}$ \\
\hline elavGS>UAS-lacZ & 181 & $62.4 \pm 1.1$ & $/$ & $/$ \\
\hline elavGS>UAS- $h$ TDP-43 + & & & $/$ \\
\hline RNAi-mCherry & 184 & $43.9 \pm 1.3$ & $/$ & $/$ \\
RNAi-Parkin & 137 & $45.4 \pm 0.9$ & $\mathrm{~ns}$ & $+11.2 \%$ \\
RNAi-PINK1 & 179 & $48.8 \pm 0.9$ & $\star \star$ & $/ 14.8 \%$ \\
\hline UAS-lacZ & 137 & $38.6 \pm 0.7$ & $/$ & $/$ \\
UAS-Parkin & 165 & $44.3 \pm 0.7$ & $* \star$ & $\mathrm{ns}$ \\
UAS-PINK1 & 139 & $39.4 \pm 1.4$ & &
\end{tabular}

Fig. 7 Parkin and PINK1 show distinct modifying effects in a Drosophila model of TDP-43 proteinopathy. $\mathbf{a}$, $\mathbf{b}$ The effect of KD (a) or OE (b) of Parkin or PINK1 on hTDP-43 flies in climbing assays. RNAi-mCherry is used as a control for RNAi-Parkin and RNAi-PINK1, and UAS-lacZ is a control for UAS-Parkin and UAS-PINK1. Transgenic expression is restricted to the adult neurons using an elavGS driver (induced with RU486, $80 \mu \mathrm{g} / \mathrm{ml}$, starting from day 1 of the adulthood). The locomotive capability is evaluated as the average percentage of flies that climb over $3 \mathrm{~cm}$ within $10 \mathrm{~s}$. Data are shown as mean $\pm \mathrm{SEM}, n=20$ flies/vial and 5 vials for each group. c-e PINK1 KD or Parkin OE in the adult neurons significantly extends the lifespan of hTDP-43 flies. The lifespan assay of TDP-43 flies with adult-onset neuronal KD (c) or OE (d) of Parkin or PINK1. elavGS > UAS-lacZ is used as a nondisease UAS transgene control for UAS-hTDP-43. e Summary of the median lifespans, shown as mean \pm SEM; $n$, the number of flies tested for each genotype is indicated; ${ }^{* *} p<0.01,{ }^{* * *} p<0.001$; ns not significant; Student's $t$-test

showed minimal ${ }^{31}$ or no statistically significant increase of longevity (Fig. S5A-B). Thus, the improvement of TDP-43 flies' survival was unlikely a generic effect of Parkin OE or PINK1 KD on aging. Rather, the results suggested a specific involvement of Parkin and PINK1 in TDP-43 proteinopathy.

\section{Discussion}

Mitochondrial dysfunction has been linked to the pathogenesis of ALS $^{32-36}$. Parkin and PINK1 are both important genes involved in mitochondrial quality control, and previous sequencing studies identified Parkin pre-mRNA as a RNA target of TDP- $43^{12,13}$. Interestingly, 
Parkin mRNA levels were decreased in the brain of TDP43 KD mice; whereas in sALS patient cells, Parkin protein level decrease was associated with excessive TDP-43 accumulation $^{12}$. This is likely because TDP-43-mediated neurodegeneration involves both LOF and GOF mechanisms ${ }^{23}$. In this study, we focus on how TDP-43 GOF affects Parkin and PINK1, and use multiple cell and animal models of TDP-43 proteinopathy to demonstrate that they are differentially misregulated at RNA and protein levels by TDP-43 OE.

In all models tested in this study, we observe a significant reduction of Parkin mRNA and protein levels (Figs. 1 and 2). Consistently, the increase of Parkin suppresses the degenerative phenotypes of hTDP-43 flies (Fig. 7) and reduces neuronal cell death in the motor cortex of rat TDP-43 model ${ }^{37}$. We speculate that the TDP-43 KD-induced Parkin mRNA reduction may be due to loss of TDP-43 binding and protection of the Parkin pre-mRNA ${ }^{12,13}$, whereas TDP-43 OE-induced Parkin decrease may result from abnormal binding and misregulation of mature Parkin mRNA (Figs. 3 and 4) or other mechanisms to be discovered. Nevertheless, such seemingly contradictory effects are also observed with TDP-43 in regulating alternative splicing. Taking the TDP-43 targets Kcnd3 and Ahi1 as an example, both KD and OE of TDP- 43 cause aberrant alternative splicing of them in the same direction ${ }^{24}$.

As a RNA-binding protein, TDP-43 regulates various aspects of RNA metabolism, including the sustenance of long intron-containing pre-mRNA, alternative splicing, $3^{\prime}$ UTR-mediated mRNA transport or stability, and regulation of long noncoding RNAs ${ }^{2,12,13,15}$. All of these regulations appear to require noncoding sequences such as introns and $3^{\prime}$ UTRs. One intriguing finding of this study is that TDP-43 not only regulates the mRNA of long intron-containing human and mouse Parkin but also reduces the mRNA levels of Parkin transcribed from the dParkin gene with only very short introns. We further exemplify that in the absence of any intron or UTR, plasmid-expressed hParkin can still be downregulated by TDP-43 (Fig. 3), which suggests an additional, intronindependent regulation of mRNA by TDP-43.

Furthermore, we find that downregulation of intronfree hParkin requires the RRM1 and GRD domains of TDP-43 (Fig. 4), indicating that this regulation depends on both the RNA-binding and the protein-protein interaction of TDP-43. It is reasonable to speculate that TDP-43 may bind to the coding region of Parkin via the RRM1 motif and recruit other proteins that regulate mRNA stability via the GRD domain. Alternatively, TDP-43 might indirectly decrease Parkin mRNA levels by regulating other RNA-binding proteins. Lending support to this possibility, TDP-43 regulates the RNA splicing of the PUF-domain-containing protein PUM1, which regulates mRNA stability ${ }^{38}$. Also, we notice that the coding region of Parkin contains a TGTAAAGA sequence, which encodes the mRNA that is only one nucleotide different from the PUF-binding sequence (UGUANAUA) and might be recognized by PUM1 ${ }^{39}$. However, as the PUF recognition sequence usually locates within $3^{\prime}$ UTRs, the exact mechanism of this enigmatic intron/UTR-independent regulation of Parkin mRNA is yet to be unraveled by additional research in the future.

Although TDP-43 does not directly regulate PINK1 mRNA or protein levels, we find that it selectively impedes PINK1 protein turnover and causes cleaved PINK1 to accumulate in the cytoplasm due to the impairment of proteasomal degradation (Fig. 5 and Fig. S4). This alteration may cause cytotoxicity at two levelson one side, it may reduce PINK1 interaction with its normal mitochondrial substrates such as $\mathrm{NdufA} 10^{40}$, leading to mitochondrial dysfunction; on the other side, the cytosolic accumulation of PINK1 may cause gain of toxicity due to ectopic or increased phosphorylation of cytosolic substrates such as Parkin, ubiquintin, and others yet to identify ${ }^{41,42}$. Along the line, although we did not observe substantial induction of mitophagy by TDP-43 $\mathrm{OE}$ in our systems (Fig. S7), it is reported that cytosolic accumulation of cleaved PINK1 induces non-selective mitophagy ${ }^{43}$ and constitutive activation of PINK1 triggers non-apoptotic cell death that is independent of mitophagy ${ }^{44}$. In this study, we show that the increase of cleaved PINK1 reduces the reserve respiration capacity of mitochondria (Fig. 6), which may contribute to disease progression in TDP-43 proteinopathy.

The misregulation of the Parkin-PINK1 pathway shall have profound detrimental consequences. LOF mutations of the Parkin and PINK1 genes are associated with juvenile $\mathrm{PD}^{19,20}$. In flies, Parkin and PINK1 LOF mutants exhibit mitophagy defects and cell death in muscles, which subsequently cause locomotion defects and reduce the longevity ${ }^{45-48}$. Moreover, mitochondrial fragmentation has been observed in transgenic mouse and fly models of TDP- $43^{33,34,49}$ as well as fibroblasts obtained from patients carrying TDP-43 mutations ${ }^{50}$. Since the Parkin-PINK1 pathway also regulates mitochondrial fission-fusion proteins ${ }^{51,52}$, their misregulation may contribute to TDP-43-induced mitochondrial over-fission. Similarly, the substrates of Parkin range from proteins regulating mitochondrial dynamics, transport, components of the electron transport chain, to proteins functioning in the proteasome and the nucleus ${ }^{53}$. Therefore, the TDP-43-mediated downregulation of Parkin is likely to have comprehensive deleterious effects.

In summary, in this study we find that Parkin and PINK1 are differentially misregulated in TDP-43 proteinopathy (Fig. 8). TDP-43 OE reduces Parkin mRNA 


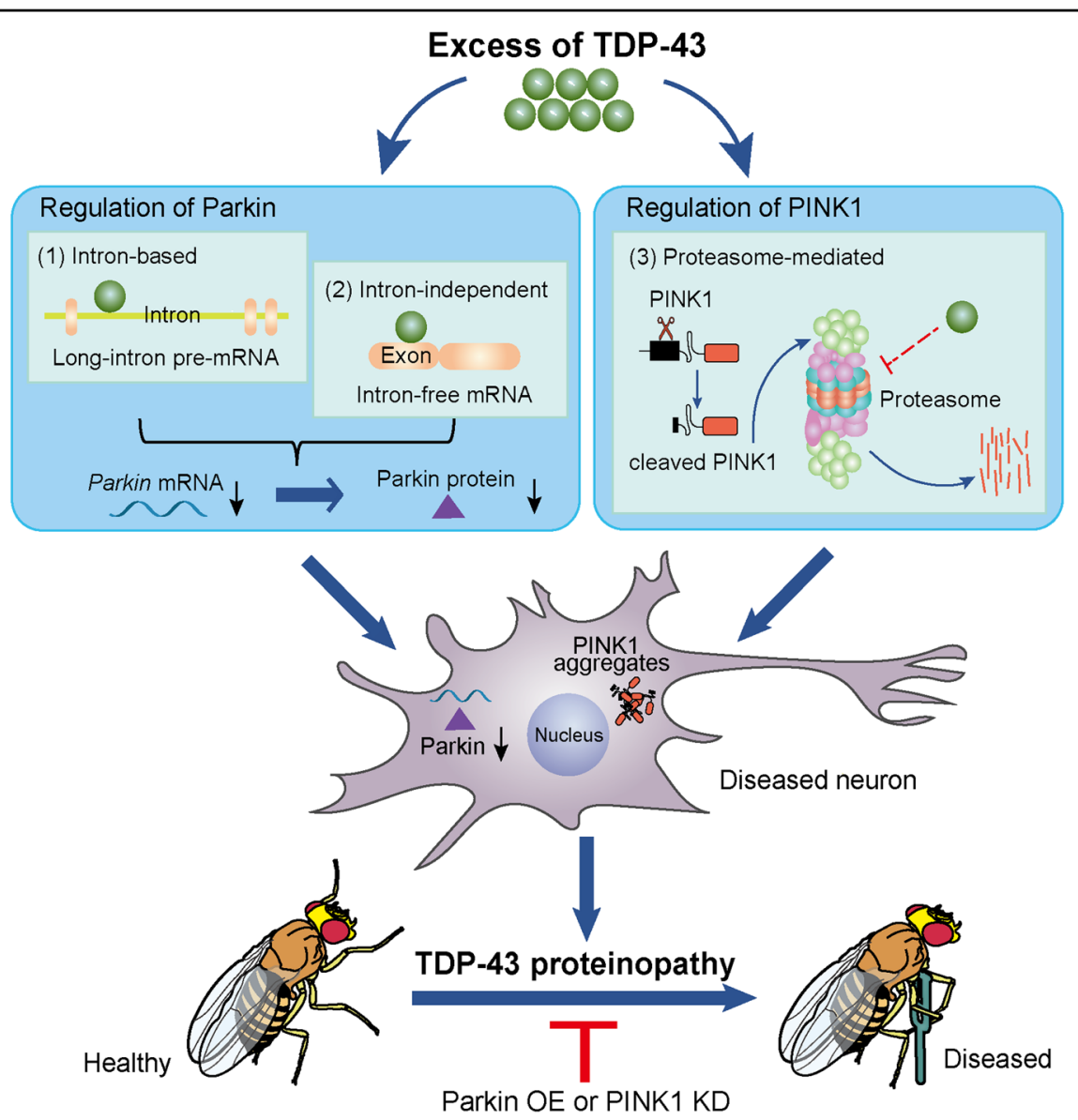

Fig. 8 A schematic model of the multilevel misregulation of Parkin and PINK1 in TDP-43 proteinopathy. TDP-43 protein inclusion is a pathological hallmark of ALS and other neurodegenerative diseases of TDP-43 proteinopathies. In this study, we reveal that TDP-43 OE misregulates the mitochondrial quality control genes Parkin and PINK1 differently and at multiple levels. In addition to (1) the previously reported TDP-43 function in regulating long intron-containing pre-mRNA of Parkin, we find that (2) Parkin mRNA without any intron can also be robustly decreased by TDP-43, suggesting an unidentified, intron-independent mechanism. The intron-based and the intron-independent regulations together lead to a significant reduction of Parkin protein abundance. Meanwhile, (3) excessive TDP-43 impairs proteasomal activity that impedes the turnover of cleaved PINK1, resulting in cytosolic accumulation of insoluble PINK1 aggregates. Consistent with the changes at the cellular level, Parkin OE but PINK1 KD in a Drosophila model of TDP-43 significantly suppresses the degenerative phenotypes

levels, which involves both intron-based and intronindependent mechanisms. In the meantime, excessive TDP-43 impairs the proteasomal activity, which hinders PINK1 turnover and causes cytosolic accumulation of cleaved PINK1. Consistently, upregulation of Parkin, whereas downregulation of PINK1 suppresses TDP-43induced neurodegeneration in a Drosophila model of ALS. Together, we propose that Parkin and PINK1 are differentially misregulated at RNA and protein levels, which may contribute to the pathogenesis of TDP-43 proteinopathy. As functions of Parkin and PINK1 beyond regulating mitophagy or mitochondria are increasingly revealed, our findings strongly suggest that differential therapeutic strategies need to be developed when considering the Parkin-PINK1 pathway as a common target for treating ALS.

\section{Materials and methods \\ Plasmids and constructs}

To generate the pCAG-Flag-Parkin, pCAG-PINK1-V5, and pCAG-hTDP-43-HA plasmids, DNA fragments encoding human Parkin, PINK1, and TDP-43 were amplified from YFP-PARK2 (Addgene \#23955 ${ }^{54}$ ), pcDNA-DEST47 PINK1 C-GFP (Addgene \#13316 ${ }^{55}$ ), and pcDNA3.1-TDP-43-Myc plasmid (a gift from $\mathrm{H}-\mathrm{Y} \mathrm{Hu}$ ) by PCR using primers containing the Flag, V5, or HA-tag sequence. The PCR products were then subcloned into a pCAG vector ${ }^{56}$ using the $X h o I / E c o R I$ sites. The pDsRed2mito vector was from Clontech Laboratories, Inc.

The expression constructs of truncated TDP-43 were generated by homologous recombination. Briefly, the DNA fragments of truncated hTDP-43-HA were amplified by PCR and inserted into the cloning vector using 
ClonExpress MultiS One Step Cloning Kit (Vazyme). The constructs were then subcloned into the pCAG expression vector as above. The PCR primers used were listed below:

$\triangle$ RRM1-F1: 5'-ATGGCCATGGAGGCCGAATTCAT GTCTGAATATATT-3'

$\triangle$ RRM1-R1: 5'-CTCATCTTGGCTTTGGGATGTTT

TCTGGACTGCTC-3'

ARRM1-F2: 5'-CAAAGCCAAGATGAG-3'

$\triangle$ RRM1-R2: $\quad 5^{\prime}$-GATGAGATGTTTTCTGGACTGC

TC-3'

ARRM2-F1: 5'-ATGGCCATGGAGGCCGAATTCAT GTCTGAATATATT-3'

ARRM2-R1: 5'-CTATTGCTATTGTGCTTAGGGC TTCTCAAAGGCTCATCTT-3'

ARRM2-F2: 5' -CCTAAGCACAATAGC-3'

$\triangle$ RRM2-R2: 5'-CATGTCTGGATCCCCGCGGCCG CCTACATTCCCCAGCCAGA-3'

$\triangle$ GRD-F: 5 '-ATGGCCATGGAGGCCGAATTCATG TCTGAATATATT-3'

$\triangle$ GRD-R: 5 '-CATGTCTGGATCCCCGCGGCCGCCT

AACTTCTTTCTAACTGTCTATT-3'

To generate the pCAG-PINK $1^{\Delta 1-104}-\mathrm{V} 5$ plasmid, the DNA fragments of truncated PINK1 were amplified from FL PINK1 by PCR and inserted into the PCAG vector as above. The PCR primers used were:

PINK1 ${ }^{\Delta 1-104}$-F: $\quad 5^{\prime}$-CATCATTTTGGCAAAGAATTC CACCATGGGGCTAGGGCTGGGCCTC-3'

PINK1 ${ }^{\Delta 1-104}$-V5-R: $\quad 5^{\prime}$-GCTCCCCGGGGGTACCTCG AGTTACGTAGAATCGAGACCGAGGAGAGGGTTAG GGATAGGCTTACCCAGGGCTGCCCTCCATGAG-3'.

All constructs were verified by sequencing to ensure the integrity of the cloned open reading frames.

\section{Cell cultures and transfection}

293T cells were cultured in Dulbecco's modified Eagle medium (Invitrogen) supplemented with $10 \%(\mathrm{v} / \mathrm{v})$ fetal bovine serum (BioWest) and GlutaMAX ${ }^{\mathrm{TM}}$ (Invitrogen) at $37^{\circ} \mathrm{C}$ in $5 \% \mathrm{CO}_{2}$. Transient transfection was performed using Lipofectamine 2000 (Invitrogen) in Opti-MEM (Invitrogen). Cells were transfected for $48 \mathrm{~h}$ before harvest. For proteasome inhibition assays, MG-132 was added into the medium $3 \mathrm{~h}$ before harvest at a final concentration of $50 \mathrm{nM}$.

\section{Lentivirus production and primary neuron culture}

Lentivirus was prepared according to the established protocols. Briefly, lentivirus packaging was performed by co-transfecting FHsynPW-TDP-43-HA with VSVG and delta 8.9 with the ratio of 1:1.5:2 into 293FT cells cultured in Opti-MEM I medium using PolyJet ${ }^{\mathrm{mw}}$ reagent (SignaGen). Culture supernatant was collected at $48 \mathrm{~h}$ after transfection and passed through a $0.45-\mu \mathrm{m}$ filter. Viral particles were concentrated from culture supernatants by Lenti- $\mathrm{X}^{\mathrm{mu}}$ Concentrator (Clontech). Viral pellets used for neuronal infection were resuspended in Neurobasal medium (Invitrogen).

Primary hippocampal neurons were isolated from C57BL/6 mouse hippocampus at embryonic day 14 (E14) and cultured in serum-free Neurobasal medium (Invitrogen) supplemented with 2\% B27 (Life Technologies), GlutaMax, and penicillin-streptomycin (Invitrogen). At 14 days in vitro (DIV), neurons were infected with FHsynPW-TDP-43-HA for 5 days before extraction for RNA or protein. For TDP- $43^{\mathrm{Q} 331 \mathrm{~K}}$ mouse-derived culture, the cortical neurons of the transgenics or their littermates were isolated at E16-E18 and cultured as above. The RNA and protein levels of TDP- $43^{\mathrm{Q} 331 \mathrm{~K}}$ neurons were examined at 15 DIV.

\section{TDP-43 transgenic mice}

The TDP- $43^{\mathrm{Q} 331 \mathrm{~K}}$-myc transgenic mice ${ }^{24}$ were derived from the B6N.Cg-Tg (Prnp-TARDBP*Q331K) 103Dwc/J line (Jackson Laboratory, \#017933) and maintained by breeding to the C57BL/6N background mice purchased from Vital River Laboratories Co., Ltd. All animal experiments were carried out in compliance with the institutional guidelines on the scientific use of living animals at Interdisciplinary Research Center on Biology and Chemistry (IRCBC) of the Chinese Academy of Sciences (Protocol \#: IRCBC-2016-016, IRCBC Animal Care and Use Committee).

\section{Drosophila strains}

The following strains were obtained from the Bloomington Drosophila Stock Center: RNAi-mCherry (\#35785, a control for in vivo RNAi knockdown); UASlacZ (\#8529, a control for UAS transgene expression); elavGS (\#43642); UAS-PINK1 (\#51648); and UAS-Parkin (\#51651). The following strains were obtained from the Tsinghua Fly Center: RNAi-PINK1 (\#38262) and RNAiParkin (\#38333). The transgenic fly strain of UAS-TDP43 was generated by $Ф C 31$ integrase-mediated, sitespecific integration, and the attP landing site stock used was UAS-phi2b2a;VK5 (75B1).

Flies tested in this study were raised on standard cornmeal media and maintained at $25^{\circ} \mathrm{C}$ and $60 \%$ relative humidity. For adult-onset, neuronal expression of the UAS or RNAi transgenes using the elavGS driver, ${ }^{59}$ flies were raised on regular fly food supplemented with $80 \mu \mathrm{g} / \mathrm{ml} \mathrm{RU} 486$ (TCI).

\section{Generation of the dParkin-(HA) KI fly line Construction of the pCR2-TOPO-2xHA-PBac-3xp3-eGFP vector}

A 2xHA tag donor vector was generated from the pCR2-TOPO-3xp3-eGFP HR vector following the protocols on the flyCRISPR website. The 2xHA-stop 
codon-fused $\mathrm{pBac}$ 3'-terminal repeat fragments were created based on the pHD-2xHA-ScarlessDsRed vector by PCR reaction using the following primers: F1_5'ACGTGC

CAGACTATGCTTAACCCTAGAAAGATAATCATAT TGTGACGTAC-3', F2_5'-ATGTTCCAGATTACGCTG GATATCCATACGACGTGCCAGACTATGCTTAA-3', F3_5'-gctagcctgacccgggGAAGACCTTACCCATACGAT GTTCCAGATTACGCTGGA-3' R_5'-aattagatcccctagg TAAAAGTTTTGTTACTTTATAGAAGAAATT- $3^{\prime}$.

A 338-bp PCR product was generated and cloned into the NdeI/AvrII sites of pCR2-TOPO-3xp3-eGFP HR vector by In-Fusion HD Cloning Kit (Clontech) to get a transition vector. The $\mathrm{pBac} 5^{\prime}$-terminal repeat fragment was created from pHD-2xHA-ScarlessDsRed by PCR reaction using the following primers:

F_5'-cttatcgaatacgcgtGATATCTATAACAAGAAAAT ATATATATAA-3' R_5'-ctcgagcgatcatatgCGTCTCCT TAACCCTAGAAAGATAGTCTGCGTAAAAT-3'.

A 352-bp PCR product was generated and cloned into the MluI/NdeI sites of the transition vector by In-Fusion HD Cloning Kit to get the pCR2-TOPO-2xHA-PBac3xp3-eGFP vector.

\section{Construction of the pCR2-TOPO-dParkin-2xHA-PBac-3xp3- eGFP HR donor vector}

The L-arm of the dParkin flanking sequence without the stop codon was generated by PCR of the genomic DNA from the $\mathrm{W}^{1118}$ flies with the following primers:

$\mathrm{F}_{-} \quad 5^{\prime}$-atccactagtgctagcGGATTTTGCGGCTCCATTG AATCTAATCAA-3'

R_5'-aacatcgtatgggtaGCCGAACCAGTGAGCTCCCA TGCAGTCGCG-3'.

A 1032-bp PCR product was generated and cloned into the NheI/BbsI sites of pCR2-TOPO-2xHA-PBac-3xp3eGFP by In-Fusion HD Cloning Kit to get a Parkin-2xHA transition vector. The $\mathrm{R}$-arm of $d$ Parkin flanking sequence was generated similarly using the following primers:

F_5'-tctttctagggttaaTCCTTCGTCATCGTTGTACGT CGGCCCGACGTGTCCTTTTTG-3'

R_5'-tagatgcatgctcgagAGCGGCAAACCGAAAGGAA TCTAGAACGTG-3'.

A 1149-bp PCR product was generated and cloned into the $B s m b I / X h o I$ sites of dParkin-2xHA transition vector by In-Fusion HD Cloning Kit to get the complete pCR2TOPO-dParkin-2xHA-PBac-3xp3-eGFP HR donor vector.

\section{Construction of the pBFv-U6.3-dParkin-stop gRNA vector}

The target sites of dParkin gene for CRISPR/Cas9 recognition were designed using the flyCRISPR Optimal Target Finder platform of flyCRISPR website. Two target sites close to the stop codon of the dParkin gene were found: gRNA-t1 site 5'-GAAGGATTAGCCGAACC
AGTGGG-3' and gRNA-t2 site 5'-TCGTTGTACG TCGGCCCGACGGG-3'. The target site fragments were generated by primer annealing and cloned into the $B b s$ sites of pBFv-U6.3 vector (modified from $\mathrm{pBFv}-\mathrm{U} 6.2$ ) to get the pBFv-U6.3-dParkin-stop gRNA-t1 and gRNA-t2 plasmids.

\section{Embryo injection and transformant selection}

The two pBFv-U6.3-dParkin-stop gRNA plasmids were mixed with the pCR2-TOPO-dParkin-2xHA-PBac-3xp3eGFP HR donor vector for nanos-Cas9 founder line injection. Following green fluorescent protein (GFP)mediated identification of successful KI flies, the GFP marker was removed through a single cross to PBac transposase. The embryo injection and selection of correct transformants were performed by BestGene Inc. and confirmed by PCR in the lab.

\section{RNA extraction and real-time quantitative PCR}

For quantitative PCR (qPCR), total RNA was isolated from fly heads, cell cultures, or mouse brain tissues using TRIzol (Invitrogen) according to the manufacturer's instruction. After DNase (Promega) treatment to remove genomic DNA, the RT reactions were performed using All-in-One cDNA Synthesis SuperMix kit (Bimake). The cDNA was then used either for semiquantitative RTPCR experiments by PCR amplification using the Taq Plus Master Mix (Vazyme) or real-time qPCR using the SYBR Green qPCR Master Mix (Bimake) with the QuantStudio $^{\text {Ta }} 6$ Flex Real-Time PCR system (Life Technologies). The mRNA levels of actin or GAPDH were used as an internal control to normalize the mRNA levels of genes of interest. The qPCR primers used in this study are listed below:

h $\beta$-actin forward: 5'-GTTACAGGAAGTCCCTTGCC ATCC-3'

h $\beta$-actin reverse: 5'-CACCTCCCCTGTGTGGACTT GGG-3'

hPINK1 forward: 5'-CCCAAGCAACTAGCCCCTC-3'

hPINK1 reverse: 5'-GGCAGCACATCAGGGTAGTC-3'

hParkin forward: 5'-GTGTTTGTCAGGTTCAACT CCA-3'

hParkin reverse: 5'-GAAAATCACACGCAACTGGT C-3'

hTDP-43 forward: 5'-GGGAAATCTGGTGTATGTT GTCA-3'

$h T D P-43$ reverse: 5'-GAAAATCACACGCAACTGG TC-3'

PINK1-V5 forward: 5'-CAGACGTGAGACAGTTG GTG-3'

PINK1-V5 reverse: 5'-GTAGAATCGAGACCGAGGA GAG-3'

Flag-Parkin forward: 5'-CAAGGATGACGACGATAA GT-3'

Flag-Parkin reverse: 5'-GCTGGAAGATGCTGGTGT-3' 
$m G A P D H$ forward: 5'-CACCATCTTCCAGGAGCG AG-3'

$m G A P D H$ reverse: 5'-CCTTCTCCATGGTGGTGAA

GAC-3'

mPINK1 forward: 5'-CACACTGTTCCTCGTTATG

AAGA-3'

mPINK1 reverse: 5'-CTTGAGATCCCGATGGGCA

AT-3'

mParkin forward: $5^{\prime}$-TCTTCCAGTGTAACCACCG

TC-3'

mParkin reverse: 5'-TCTTCCAGTGTAACCACCGTC-3'

dActin forward: 5'-GAGCGCGGTTACTCTTTCAC-3' dActin reverse: 5'-GCCATCTCCTGCTCAAAGTC-3' dPINK1 forward: 5'-GAGCAACAGCAGCATCAGAA-3' dPINK1 reverse: 5'-TGATGTTTGAATTCGCTGGA-3' dParkin forward: 5'-AGCGATGCCACGACAATAG AGC-3'

dParkin reverse: 5'-GCGAAGGTTCCTCCTCCTCC AA-3'

\section{Antibodies}

The following antibodies were used for western blotting, immunoprecipitation, and immunofluorescence assays: mouse anti-FLAG (Sigma-Aldrich, F3165); mouse anti-HA (Proteintech, 66006-1); rabbit anti-HA (CST, 3724); rabbit anti-TDP-43 (Proteintech, 10782-2-AP); rabbit anti-PINK1 (Novus Biologicals, BC100-494); mouse anti-Parkin (Santa Cruz, sc-32282); mouse anti-V5 (Proteintech, 66007-1); mouse anti-GAPDH (Proteintech, 60004-1); rabbit antiTubulin (MBL, PM054); mouse anti-Lamin C (DSHB, LC28.26); and chicken anti-MAP2 (Abcam, ab5392). Horseradish peroxidase-conjugated secondary antibodies: anti-mouse (Sigma-Aldrich, A4416) and anti-rabbit (SigmaAldrich, A9169). Fluorescent secondary antibodies: antimouse Cy5 (Life Technologies, A10524), anti-rabbit Alexa Fluor 488 (Life Technologies, A11012), and anti-chicken Alexa Fluor 633 (Sigma-Aldrich, A-21103).

\section{Protein extraction}

Fly heads or cultured cells were lysed in $2 \%$ SDS lysis buffer $(100 \mathrm{mM}$ Tris- $\mathrm{HCl}$ at $\mathrm{pH} 6.8,2 \%$ SDS, $40 \%$ glycerol, $10 \% \beta$-mercaptoethanol, and $0.04 \%$ bromophenol blue) containing protease and phosphatase inhibitor cocktails (Roche). For separation of soluble and insoluble proteins, cells were lysed on ice using RIPA buffer (50 mM Tris at pH 8.0, $150 \mathrm{mM} \mathrm{NaCl}, 1 \%$ NP-40, $5 \mathrm{mM}$ EDTA, 0.5\% sodium deoxycholate, and $0.1 \%$ SDS) supplemented with protease and phosphatase inhibitors (Roche). Samples were sonicated and then centrifuged at $13000 \times g$ for $20 \mathrm{~min}$ at $4{ }^{\circ} \mathrm{C}$. The resulting supernatant was used as the soluble fraction and the pellets containing insoluble fractions were dissolved in a $9 \mathrm{M}$ urea buffer ( $9 \mathrm{M}$ urea, $50 \mathrm{mM}$ Tris buffer, $\mathrm{pH} 8.0$ ) after wash.
Fresh brains of deep-anesthetized mice were rapidly excised and the motor cortices were isolated and collected in sterile $1.5 \mathrm{ml}$ micro-centrifuge tubes. The samples were quickly plunged into liquid nitrogen and stored at $-80^{\circ} \mathrm{C}$ until testing. The brain tissues were then lysed and homogenized in ice-cold RIPA buffer supplemented with protease and phosphatase inhibitors (Roche).

\section{Western blotting}

Equivalent amounts of lysates were resolved by electrophoresis through a $10 \%$ Bis-Tris SDS-polyacrylamide gel electrophoresis gel (Invitrogen) and probed with the primary and secondary antibodies listed above. Detection was performed using the High-sig ECL Western Blotting Substrate (Tanon). Images were captured using an Amersham Imager 600 (GE Healthcare) and densitometry was measured using ImageQuant TL Software (GE Healthcare). The contrast and brightness were optimized equally using Adobe Photoshop CS6 (Adobe Systems Inc.). All experiments were normalized to GAPDH, tubulin, or actin levels as indicated in the blots and the values are plotted relative to the control (set to a value of 1) in across-assay comparison quantifications.

\section{Nuclear and cytoplasmic extraction}

For nuclear-cytoplasmic fractionation, 20 flies per genotype were homogenized in the lysis buffer [ $50 \mathrm{mM}$ Tris at $\mathrm{pH} 7.4,10 \mathrm{mM} \mathrm{NaCl}, 0.5 \% \mathrm{NP}-40,0.25 \%$ Triton X100, $1 \mathrm{mM}$ EDTA, and protease inhibitors (Roche)] by incubating for $5 \mathrm{~min}$ on ice as reported ${ }^{15}$ followed by centrifuge at $3000 \times g$ for $5 \mathrm{~min}$ at $4{ }^{\circ} \mathrm{C}$. The supernatant was collected as the cytoplasmic fraction and the pellet was dissolved in tissue lysis buffer (Invitrogen) as the nuclear fraction.

\section{Immunocytochemistry and confocal imaging}

293T cells or primary neurons were grown on Nunc Chambered Coverglasses (Lab-Tek) and transfected with the indicated plasmids for $24 \mathrm{~h}$. Thereafter, cells were fixed in $4 \%$ paraformaldehyde in phosphate-buffered saline (PBS) for $15 \mathrm{~min}$ at room temperature, permeabilized with $0.5 \%$ Triton X-100 in PBS for $15 \mathrm{~min}$, and blocked with $3 \%$ goat serum in PBST (PBS $+0.1 \%$ Triton X-100) for $1 \mathrm{~h}$ at room temperature. The above primary and secondary antibodies in the blocking buffer were then incubated at $4{ }^{\circ} \mathrm{C}$ overnight or at room temperature for 1 h. After three washes with PBST, cells were mounted on glass slides using Vectashield Antifade Mounting Medium with DAPI (Vector Laboratories).

Fluorescent images were taken with Leica TCS SP8 confocal microscopy system using a $\times 63$ oil objective (numerical aperture $=1.4$ ). Co-localization of Parkin or PINK1 with mitochondria was evaluated by the line scanning analysis of LAS $\mathrm{X}$ and the protein puncta were 
counted using the "Analyze Particles" module of ImageJ. Images were assembled into figures using Adobe Photoshop CS6.

\section{Proteasomal activity assay}

Proteasomal activity was measured using a Proteasome Activity Assay kit (Abcam, ab107921). 293T cells were lysed in PBS with $0.5 \% \mathrm{NP}-40$ on ice for $10 \mathrm{~min}$. The supernatant was obtained by centrifuge at $13000 \times g$ for $10 \mathrm{~min}$ at $4{ }^{\circ} \mathrm{C}$. The proteasomal activity of the supernatant was determined by assaying the cleavage of a fluorogenic peptide substrate Suc-LLVY-AMC according to the manufacturer's instruction. The fluorescence intensity was measured after the substrate peptide was incubated with the cell lysates at $37^{\circ} \mathrm{C}$ at the end of the assay (60 $\mathrm{min}$ ) using a microplate reader (BioTek, Ex/ $\mathrm{Em}=350 / 440 \mathrm{~nm})$.

\section{OCR measurement}

Mitochondrial respiration functions were evaluated by measuring the OCRs using the Seahorse XFe96 Analyzer (Agilent) per the manufacturer's instruction. Briefly, 24 h after transfection, $4 \times 10^{4} 293 \mathrm{~T}$ cells were seeded onto 96well microplates pre-coated with poly-L-lysine. On the next day, the OCRs $(\mathrm{pmol} / \mathrm{min})$ of the cells in XF base medium containing $1 \mathrm{mM}$ pyruvate, $2 \mathrm{mM}$ glutamine, and $10 \mathrm{mM}$ glucose (Sigma-Aldrich) were assayed with the Seahorse CF Cell Mito Stress Test following sequential additions of $1 \mu \mathrm{M}$ oligomycin, $0.5 \mu \mathrm{M}$ Carbonyl cyanide4-(trifluoromethoxy)phenylhydrazone, and a combination of $0.5 \mu \mathrm{M}$ antimycin $\mathrm{A}$ and $0.5 \mu \mathrm{M}$ rotenone.

\section{Fly lifespan and climbing assays}

For the lifespan experiment, 20 flies/vial, 5-8 vials/ group were tested. Flies were transferred to fresh fly food every 3 days and the number of dead flies of each vial was recorded. Flies lost through escape or accidental death were excluded from the final analysis. The median lifespan was calculated as the mean of the medians of each vial belonging to the same group, whereas the "50\% survival" shown on the survival curves was derived from compilation of all vials of the group. For the climbing assay, 20 flies were transferred into an empty polystyrene vial and gently tapped down to the bottom of the vial. The number of flies that climbed over a distance of $3 \mathrm{~cm}$ within $10 \mathrm{~s}$ was recorded. The test was repeated three times for each vial and 5 vials of each genotype were tested. ${ }^{57,58}$

\section{Statistical analysis}

Unless otherwise noted, statistical significance in this study is determined by unpaired, two-tailed Student's $t$-test at ${ }^{*} p<0.05,{ }^{* * *} p<0.01$, and ${ }^{* * * *} p<0.001$. Error bars represent the standard error of the mean.

\section{Acknowledgements}

We thank the BDSC and the TFC for providing fly strains, A. Du for assistance in cell culture experiments, and J. Yuan, Y. Chen, and C. Liu for helpful discussion and critical reading of the manuscript. This study was supported by the National Key R\&D Program of China (No. 2016YFA0501902) and the National Natural Science Foundation of China (No. 31471017 and No. 81671254) to Y.F.; the National Natural Science Foundation of China (No. 81501171) and the Fundamental Research Funds for the Central Universities to A.L. The affiliations and addresses listed in this work represent no policital opinion of the authors.

\section{Author details}

${ }^{1}$ Interdisciplinary Research Center on Biology and Chemistry, Shanghai Institute of Organic Chemistry, Chinese Academy of Sciences, Shanghai 201210, China. ${ }^{2}$ University of Chinese Academy of Sciences, Beijing 100049, China.

${ }^{3}$ Guangdong-Hong Kong-Macau Institute of CNS Regeneration, Joint International Research Laboratory of CNS Regeneration Ministry of Education, Guangdong Medical Key Laboratory of Brain Function and Diseases, Jinan University, Guangzhou 510632, China. ${ }^{4}$ National Institute of Infectious Diseases and Vaccinology, National Health Research Institutes, Miaoli County, Taiwan

\section{Authors' contributions}

X.S., Y.D., A.L. and Y.F. designed the research; X.S., Y.D., C.Q., J.-C.L., G.D., X.D., J.N. and K.T. performed the experiments; X.S., Y.D., J.L., G.D., X.C. and C.-H.C. contributed important new reagents; X.S. and Y.D. analyzed the data and prepared the figures; X.S., Y.D., A.L. and Y.F. interpreted and discussed the results; and X.S., Y.D., K.X., A.L. and Y.F. wrote the paper. All authors read and approved the final manuscript.

Conflict of interest

The authors declare that they have no conflict of interest.

\section{Publisher's note}

Springer Nature remains neutral with regard to jurisdictional claims in published maps and institutional affiliations.

Supplementary Information accompanies this paper at (https://doi.org/ 10.1038/s41419-018-1022-y).

Received: 15 May 2018 Revised: 7 August 2018 Accepted: 3 September 2018

Published online: 20 September 2018

\section{References}

1. Kiernan, M. C. et al. Amyotrophic lateral sclerosis. Lancet 377, 942-955 (2011).

2. Ling, S. C., Polymenidou, M. \& Cleveland, D. W. Converging mechanisms in ALS and FTD: disrupted RNA and protein homeostasis. Neuron 79, 416-438 (2013).

3. Neumann, M. et al. Ubiquitinated TDP-43 in frontotemporal lobar degeneration and amyotrophic lateral sclerosis. Science 314, 130-133 (2006).

4. Arai, T. et al. TDP-43 is a component of ubiquitin-positive tau-negative inclusions in frontotemporal lobar degeneration and amyotrophic lateral sclerosis. Biochem. Biophys. Res. Commun. 351, 602-611 (2006).

5. Higashi, S. et al. Concurrence of TDP-43, tau and alpha-synuclein pathology in brains of Alzheimer's disease and dementia with Lewy bodies. Brain Res. 1184, 284-294 (2007).

6. Arai, T. et al. Phosphorylated TDP-43 in Alzheimer's disease and dementia with Lewy bodies. Acta Neuropathol. 117, 125-136 (2009).

7. Toyoshima, Y. \& Takahashi, H. TDP-43 pathology in polyglutamine diseases: with reference to amyotrphic lateral sclerosis. Neuropathology 34, 77-82 (2014).

8. Chang, X. L., Tan, M. S., Tan, L. \& Yu, J. T. The role of TDP-43 in Alzheimer's disease. Mol. Neurobiol. 53, 3349-3359 (2016).

9. Chen-Plotkin, A. S., Lee, V. M. \& Trojanowski, J. Q. TAR DNA-binding protein 43 in neurodegenerative disease. Nat. Rev. Neurol. 6, 211-220 (2010).

10. Lagier-Tourenne, C., Polymenidou, M. \& Cleveland, D. W. TDP-43 and FUS/TLS: emerging roles in RNA processing and neurodegeneration. Hum. Mol. Genet. 19, R46-R64 (2010). 
11. Ratti, A. \& Buratti, E. Physiological functions and pathobiology of TDP-43 and FUS/TLS proteins. J. Neurochem. 138, 95-111 (2016).

12. Lagier-Tourenne, C. et al. Divergent roles of ALS-linked proteins FUS/TLS and TDP-43 intersect in processing long pre-mRNAs. Nat. Neurosci. 15, 1488-1497 (2012).

13. Polymenidou, M. et al. Long pre-mRNA depletion and RNA missplicing contribute to neuronal vulnerability from loss of TDP-43. Nat. Neurosci. 14, 459-468 (2011).

14. Rot, G. et al. High-resolution RNA maps suggest common principles of splicing and polyadenylation regulation by TDP-43. Cell Rep. 19, 1056-1067 (2017).

15. Tollervey, J. R. et al. Characterizing the RNA targets and position-dependent splicing regulation by TDP-43. Nat. Neurosci. 14, 452-458 (2011).

16. Deas, E. et al. PINK1 cleavage at position $\mathrm{A} 103$ by the mitochondrial protease PARL. Hum. Mol. Genet. 20, 867-879 (2011).

17. Yamano, K. \& Youle, R. J. PINK1 is degraded through the N-end rule pathway. Autophagy 9, 1758-1769 (2013).

18. Pickrell, A. M. \& Youle, R. J. The roles of PINK1, parkin, and mitochondrial fidelity in Parkinson's disease. Neuron 85, 257-273 (2015).

19. Kitada, T. et al. Mutations in the parkin gene cause autosomal recessive juvenile parkinsonism. Nature 392, 605-608 (1998).

20. Silvestri, L. et al. Mitochondrial import and enzymatic activity of PINK1 mutants associated to recessive parkinsonism. Hum. Mol. Genet. 14, 3477-3492 (2005).

21. Cozzolino, M., Ferri, A., Valle, C. \& Carri, M. T. Mitochondria and ALS: implications from novel genes and pathways. Mol. Cell. Neurosci. 55, 44-49 (2013).

22. Muyderman, H. \& Chen, T. Mitochondrial dysfunction in amyotrophic lateral sclerosis-a valid pharmacological target? Br. J. Pharmacol. 171, 2191-2205 (2014).

23. Lee, E. B., Lee, V. M. \& Trojanowski, J. Q. Gains or losses: molecular mechanisms of TDP43-mediated neurodegeneration. Nat. Rev. Neurosci. 13, 38-50 (2012).

24. Arnold, E. S. et al. ALS-linked TDP-43 mutations produce aberrant RNA splicing and adult-onset motor neuron disease without aggregation or loss of nuclear TDP-43. Proc. Natl Acad. Sci. USA 110, E736-E745 (2013).

25. Elden, A. C. et al. Ataxin-2 intermediate-length polyglutamine expansions are associated with increased risk for ALS. Nature 466, 1069-1075 (2010).

26. Kim, H. J. et al. Therapeutic modulation of elF2alpha phosphorylation rescues TDP-43 toxicity in amyotrophic lateral sclerosis disease models. Nat. Genet. $\mathbf{4 6}$, 152-160 (2014).

27. Kondo, S. \& Ueda, R. Highly improved gene targeting by germline-specific Cas9 expression in Drosophila. Genetics 195, 715-721 (2013).

28. Bassett, A. R., Tibbit, C., Ponting, C. P. \& Liu, J. L. Highly efficient targeted mutagenesis of Drosophila with the CRISPR/Cas9 system. Cell Rep. 4, 220-228 (2013).

29. Narendra, D. P. et al. PINK1 is selectively stabilized on impaired mitochondria to activate Parkin. PLoS Biol. 8, e1000298 (2010).

30. Cao, X. et al. In vivo imaging reveals mitophagy independence in the maintenance of axonal mitochondria during normal aging. Aging Cell 16, 1180-1190 (2017)

31. Rana, A., Rera, M. \& Walker, D. W. Parkin overexpression during aging reduces proteotoxicity, alters mitochondrial dynamics, and extends lifespan. Proc. Natl Acad. Sci. USA 110, 8638-8643 (2013).

32. Chen, Y. et al. PINK1 and Parkin are genetic modifiers for FUS-induced neurodegeneration. Hum. Mol. Genet. 25, 5059-5068 (2016).

33. Khalii, B. et al. Enhancing Mitofusin/Marf ameliorates neuromuscular dysfunction in Drosophila models of TDP-43 proteinopathies. Neurobiol. Aging 54, 71-83 (2017)

34. Wang, W. et al. The ALS disease-associated mutant TDP-43 impairs mitochondrial dynamics and function in motor neurons. Hum. Mol. Genet. 22 4706-4719 (2013)

35. Xu, Y. F. et al. Wild-type human TDP-43 expression causes TDP-43 phosphorylation, mitochondrial aggregation, motor deficits, and early mortality in transgenic mice. J. Neurosci. 30, 10851-10859 (2010).
36. Yung, C., Sha, D., Li, L. \& Chin, L. S. Parkin protects against misfolded SOD1 toxicity by promoting its aggresome formation and autophagic clearance. Mol. Neurobiol. 53, 6270-6287 (2016).

37. Hebron, M., Chen, W., Miessau, M. J., Lonskaya, I. \& Moussa, C. E. Parkin reverses TDP-43-induced cell death and failure of amino acid homeostasis. J. Neurochem. 129, 350-361 (2014)

38. Xiao, S. et al. RNA targets of TDP-43 identified by UV-CLIP are deregulated in ALS. Mol. Cell. Neurosci. 47, 167-180 (2011).

39. Gerstberger, S., Hafner, M.r Ascano, M. \& Tuschl, T. Evolutionary conservation and expression of human RNA-binding proteinsand their role in human genetic disease. Adv. Exp. Med. Biol. 825, 1-55 (2014).

40. Morais, V. A. et al. PINK1 loss-of-function mutations affect mitochondrial complex I activity via NdufA10 ubiquinone uncoupling. Science 344, 203-207 (2014).

41. Kane, L. A. et al. PINK1 phosphorylates ubiquitin to activate Parkin E3 ubiquitin ligase activity. J. Cell Biol. 205, 143-153 (2014).

42. Koyano, F. et al. Ubiquitin is phosphonylated by PINK1 to activate parkin. Nature 510, 162-166 (2014).

43. Lim, G. G. et al. Cytosolic PTEN-induced putative kinase 1 is stabilized by the NF-kappaB pathway and promotes non-selective mitophagy. J. Biol. Chem 290, 16882-16893 (2015).

44. Akabane, S. et al. Constitutive activation of PINK1 protein leads to proteasomemediated and non-apoptotic cell death independently of mitochondria autophagy. J. Biol. Chem. 291, 16162-16174 (2016).

45. Clark, I. E. et al. Drosophila pink1 is required for mitochondrial function and interacts genetically with parkin. Nature 441, 1162-1166 (2006).

46. Greene, J. C. et al. Mitochondrial pathology and apoptotic muscle degeneration in Drosophila parkin mutants. Proc. Natl Acad. Sci. USA 100, 4078-4083 (2003).

47. Park, J. et al. Mitochondrial dysfunction in Drosophila PINK1 mutants is complemented by parkin. Nature 441, 1157-1161 (2006).

48. Cha, G. H. et al. Parkin negatively regulates JNK pathway in the dopaminergic neurons of Drosophila. Proc. Natl Acad. Sci. USA 102, 10345-10350 (2005).

49. Magrane, J., Cortez, C., Gan, W. B. \& Manfredi, G. Abnormal mitochondrial transport and morphology are common pathological denominators in SOD1 and TDP43 ALS mouse models. Hum. Mol. Genet. 23, 1413-1424 (2014).

50. Onesto, E. et al. Gene-specific mitochondria dysfunctions in human TARDBP and C9ORF72 fibroblasts. Acta Neuropathol. Commun. 4, 47 (2016).

51. Poole, A. C. et al. The PINK1/Parkin pathway regulates mitochondrial morphology. Proc. Natl Acad. Sci. USA 105, 1638-1643 (2008).

52. Yu, W., Sun, Y., Guo, S. \& Lu, B. The PINK1/Parkin pathway regulates mitochondrial dynamics and function in mammalian hippocampal and dopaminergic neurons. Hum. Mol. Genet. 20, 3227-3240 (2011).

53. Sarraf, S. A. et al. Landscape of the PARKIN-dependent ubiquitylome in response to mitochondrial depolarization. Nature 496, 372-376 (2013).

54. Narendra, D., Tanaka, A.s Suen, D. F. \& Youle, R. J. Parkin is recruited selectively to impaired mitochondria and promotes their autophagy. J. Cell Biol. 183, 795-803 (2008)

55. Beilina, A. et al. Mutations in PTEN-induced putative kinase 1 associated with recessive parkinsonism have differential effects on protein stability. Proc. Natl Acad. Sci. USA 102, 5703-5708 (2005).

56. Chen, Y. et al. Activity-induced Nr4a1 regulates spine density and distribution pattern of excitatory synapses in pyramidal neurons. Neuron $\mathbf{8 3}, 431-443$ (2014).

57. Hebron, M. L. et al. Parkin ubiquitinates Tar-DNA binding protein-43 (TDP-43) and promotes its cytosolic accumulation via interaction with histone deacetylase 6 (HDAC6). J. Biol. Chem. 288, 4103-4115 (2013).

58. Wenqiang, C. et al. Parkin-mediated reduction of nuclear and soluble TDP-43 reverses behavioral decline in symptomatic mice. Hum. Mol. Genet. 23, 4960-4969 (2014).

59. T. Osterwalder, K. S. Yoon, B. H. White, H. Keshishian, A conditional tissuespecific transgene expression system using inducible GAL4. Proceedings of the National Academy of Sciences 98, 12596-12601 (2001) 